DRAFT VERSION APRIL 17, 2018

Typeset using LATEX twocolumn style in AASTeX61

\title{
ABSOLUTE DISTANCES TO NEARBY TYPE IA SUPERNOVAE VIA LIGHT CURVE FITTING METHODS
}

\author{
J. Vinkó,,$^{1,2,3}$ A. Ordasi, ${ }^{1}$ T. Szalai ${ }^{2}$ K. SÁrneCzKy, ${ }^{1}$ E. BÁnyai, ${ }^{1}$ I. B. Bíró, ${ }^{4}$ T. Borkovits $,{ }^{4,} 1,5$ T. HegedÜs, ${ }^{4}$ \\ G. Hodosán, ${ }^{1,5,6}$ J. Kelemen, ${ }^{1}$ P. Klagyivik,,${ }^{5,1,7,8}$ L. Kriskovics, ${ }^{1}$ E. Kun, ${ }^{9}$ G. H. Marion, ${ }^{3}$ G. Marschalkó, ${ }^{5}$ L. Molnár, ${ }^{1}$ \\ A. P. Nagy, ${ }^{2}$ A. PÁl, ${ }^{1}$ J. M. Silverman,${ }^{3}{ }^{10}$ R. Szakáts, ${ }^{1}$ E. Szegedi-Elek, ${ }^{1}$ P. Székely, ${ }^{9}$ A. Szing, ${ }^{4}$ K. Vida, ${ }^{1}$ And \\ J. C. WHEELER ${ }^{3}$ \\ ${ }^{1}$ Konkoly Observatory of the Hungarian Academy of Sciences, Konkoly-Thege ut 15-17, Budapest, 1121, Hungary \\ ${ }^{2}$ Department of Optics and Quantum Electronics, University of Szeged, Dom ter 9, Szeged, 6720, Hungary \\ ${ }^{3}$ Department of Astronomy, University of Texas at Austin, 2515 Speedway, Austin, TX, USA \\ ${ }^{4}$ Baja Observatory of the University of Szeged, Szegedi ut KT 766, Baja, 6500, Hungary \\ ${ }^{5}$ Department of Astronomy, Eötvös Loránd University, Pazmany setany 1/A, Budapest, Hungary \\ ${ }^{6}$ Centre for Exoplanet Science, School of Physics and Astronomy University of St Andrews, St Andrews KY16 9SS, UK \\ ${ }^{7}$ Instituto de Astrofsica de Canarias, C. Va Lctea S/N, 38205 La Laguna, Tenerife, Spain \\ ${ }^{8}$ Universidad de La Laguna, Dept. de Astrofsica, 38206 La Laguna, Tenerife, Spain \\ ${ }^{9}$ Department of Experimental Physics, University of Szeged, Dom ter 9, Szeged, 6720, Hungary \\ ${ }^{10}$ Samba TV, 123 Townsend St., San Francisco, CA, USA
}

(Received; Revised; Accepted)

Submitted to PASP

\begin{abstract}
We present a comparative study of absolute distances to a sample of very nearby, bright Type Ia supernovae (SNe) derived from high cadence, high signal-to-noise, multi-band photometric data. Our sample consists of four SNe: 2012cg, 2012ht, 2013dy and 2014J. We present new homogeneous, high-cadence photometric data in Johnson-Cousins $B V R I$ and Sloan $g^{\prime} r^{\prime} i^{\prime} z^{\prime}$ bands taken from two sites (Piszkesteto and Baja, Hungary), and the light curves are analyzed with publicly available light curve fitters (MLCS2k2, SNooPy2 and SALT2.4). When comparing the best-fit parameters provided by the different codes, it is found that the distance moduli of moderately-reddened SNe Ia agree within $\lesssim 0.2 \mathrm{mag}$, and the agreement is even better $(\lesssim 0.1 \mathrm{mag})$ for the highest signal-to-noise $B V R I$ data. For the highly-reddened SN 2014J the dispersion of the inferred distance moduli is slightly higher. These SN-based distances are in good agreement with the Cepheid distances to their host galaxies. We conclude that the current state-of-the-art light curve fitters for Type Ia SNe can provide consistent absolute distance moduli having less than $\sim 0.1$ -0.2 mag uncertainty for nearby SNe. Still, there is room for future improvements to reach the desired $\sim 0.05$ mag accuracy in the absolute distance modulus.
\end{abstract}

Keywords: (stars:) supernovae: individual (SN 2012cg, SN 2012ht, SN 2013dy, SN 2014J) — galaxies: distances and redshifts 


\section{INTRODUCTION}

Getting reliable absolute distances is of immense importance in observational astrophysics. Supernovae (SNe) in particular play a central role in establishing the extragalactic distance ladder. Distances to Type Ia SNe are essential data for studying the expansion of the Universe (Riess et al. 1998; Perlmutter et al. 1999; Astier et al. 2006; Riess et al. 2007; Wood-Vasey et al. 2007; Kessler et al. 2009; Guy et al. 2010; Conley et al. 2011; Betoule et al. 2014; Rest et al. 2014; Scolnic et al. 2014). SNe Ia are also especially important objects for measuring the Hubble-parameter $H_{0}$ (Riess et al. 2011, 2016; Dhawan et al. 2017), and they play a key role in testing current cosmological models (Benitez-Herrera et al. 2013; Betoule et al. 2014). Their importance has even been strengthened since the release of the cosmological parameters from the Planck mission (Planck Collaboration et al. 2014, 2015), which turned out to be slightly in tension with the current implementation of the SN Ia distance measurements (see Riess et al. 2016, and references therein).

It must be emphasized that the majority of cosmological studies use relative distances to moderate- and high-redshift SNe Ia to derive the cosmological parameters $\left(\Omega_{m}, \Omega_{\Lambda}, w\right.$, etc). From this point of view there is no need for having absolute distances, because the relative distances between different $\mathrm{SNe} / g a l a x i e s$ can be obtained with much better accuracy, especially if the galaxy is in the Hubble-flow $(z \gtrsim 0.1)$.

On the other hand, having accurate absolute distances to the nearby galaxies that are not part of the Hubble-flow is very important an astrophysical point of view. Getting reliable estimates for the physical parameters of such galaxies and the objects within them is possible only if we have reliable absolute distances on extragalactic scales.

Thus, investigating the nearest SNe Ia (within $z \lesssim 0.01$ ) can provide valuable information for various reasons. For example, distances to their host galaxies can be relatively easily determined by several methods, thus, the SN-based distances can be compared directly to those derived independently by using other types of objects and/or methods. Such very nearby galaxies might also serve as "anchors" in the cosmic distance ladder (e.g. NGC 4258, see Riess et al. 2011, 2016), which play an essential role in measuring $H_{0}$.

The popularity of SNe Ia as extragalactic distance estimators is mostly due to the fact that their absolute distances can be derived via fitting light curves (LCs) of "normal" Ia events. The LCs of such events obey the empirical Phillipsrelation, i.e. intrinsically brighter $\mathrm{SNe}$ have more slowly declining LCs in the optical bands (Pskovskii 1977; Phillips 1993). Even though nowadays SNe Ia seem to be even better standard candles in the near-infrared (NIR) regime than in the optical (Friedman et al. 2015; Shariff et al. 2016; Weyant et al. 2017), obtaining rest-frame NIR LCs for SNe Ia, except for the nearest and brightest ones, can be chal- lenging. Thus, photometric data taken in rest-frame optical bands can still provide valuable information regarding distance measurements on the extragalactic scale.

One of the main motivations of the present paper is to get absolute distances to some of the nearest and brightest recent $\mathrm{SNe}$ Ia by fitting homogeneous, high-cadence, high $\mathrm{S} / \mathrm{N}$ photometric data with public, widely-used LC-fitting codes. We selected four nearby Type Ia SNe for this project: $2012 \mathrm{cg}$, 2012ht, 2013dy and 2014J. All of them occured in the local Universe, and they were discovered relatively early (more than 1 week before B-band maximum). We have obtained new, densely sampled photometric measurements for each of them in various optical bands, which resulted in light curves extending from pre-maximum epochs up to the end of the photospheric phase. These objects, along with SN 2011fe, belong to the 10 brightest $\mathrm{SNe}$ Ia in last decade that were accessible from the northern hemisphere. However, unlike SN $2011 \mathrm{fe}$, all of them were significantly reddened by dust either in the Milky Way or in their hosts, which enabled us to test the performance of the LC-fitters in case of reddened SNe. In addition, their very low redshift $(z<0.01)$ eliminated the necessity for K-correction, which could be another possible cause for systematic errors when comparing photometry of SNe having significantly different redshifts (Saunders et al. 2015). It is also important to note that three out of four SNe in our sample have Cepheid-based distances obtained by $H S T / \mathrm{WFC} 3$, and they were recently used in calibrating $H_{0}$ with an unprecedented 2.4 percent accuracy (Riess et al. 2016).

The basic parameters for the program $\mathrm{SNe}$ are collected in Table 1.

In the following we briefly describe the observations (Section 2) and the LC-fitting codes applied (Section 3). Section 4 presents the results from the LC fitting, which are discussed further in Section 5. Section 6 summarizes the main results and conclusions.

\section{OBSERVATIONS}

Photometry of the target SNe have been carried out at two sites, located $\sim 200 \mathrm{~km}$ apart: at the Piszkéstető station of Konkoly Observatory, Hungary, and at Baja Observatory of the University of Szeged, Hungary. At Konkoly the data were taken with the $0.6 \mathrm{~m}$ Schmidt telescope through Bessell $B V R I$ filters. At Baja the observations were carried out with the $0.5 \mathrm{~m}$ BART telescope equipped with Sloan $g^{\prime} r^{\prime} i^{\prime} z^{\prime}$ filters. See Vinkó et al. (2012) for more details on these two instruments.

All data have been reduced using standard $I R A F^{1}$ routines. Transformation to the standard photometric systems

\footnotetext{
${ }^{1}$ IRAF is distributed by the National Optical Astronomy Observatories, which are operated by the Association of Universities for Research in As-
} 
Table 1. Basic data for the studied SNe

\begin{tabular}{|c|c|c|c|c|c|c|c|c|c|}
\hline SN & Discovery date & $\begin{array}{c}\mathrm{T}\left(B_{\max }\right) \\
(\mathrm{MJD})\end{array}$ & $\begin{array}{c}\Delta m_{15}(B) \\
(\mathrm{mag})\end{array}$ & Host & $z_{\text {host }}{ }^{\mathrm{a}}$ & $\begin{array}{l}D_{\text {host }}{ }^{\mathrm{b}} \\
(\mathrm{Mpc})\end{array}$ & $\begin{array}{c}E(B-V)_{M W^{\mathrm{c}}} \\
(\mathrm{mag})\end{array}$ & $\begin{array}{r}\log M_{*}{ }^{\mathrm{d}} \\
\left(M_{\odot}\right)\end{array}$ & References \\
\hline SN 2012cg & $2012-05-17$ & 56080.0 & 0.98 & NGC 4424 & 0.001458 & 16.4 & 0.018 & 9.4 & $1,9,10$ \\
\hline SN 2012ht & $2012-12-18$ & 56295.6 & 1.27 & NGC 3447 & 0.003559 & 24.1 & 0.026 & 9.3 & $2,3,9,11$ \\
\hline SN 2013dy & 2013-07-10 & 56501.1 & 0.96 & NGC 7250 & 0.003889 & 20.0 & 0.135 & 9.2 & $4,5,9,12$ \\
\hline SN 2014J & 2014-01-21 & 56689.7 & 1.03 & M82 & 0.000677 & 3.9 & 0.140 & 10.5 & $6,7,8,13$ \\
\hline
\end{tabular}

${ }^{a}$ Host galaxy redshift, adopted from NED

${ }^{b}$ Cepheid distances from Riess et al. (2016); mean redshift-independent distance from NED for SN 2014J

${ }^{c}$ Milky Way reddening based on IRAS/DIRBE maps (Schlafly \& Finkbeiner 2011)

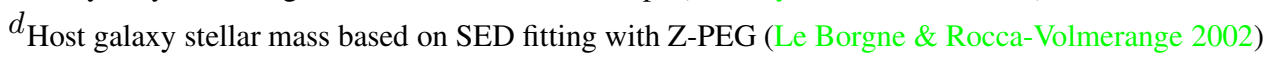

Note- References: (1):Silverman et al. (2012); (2):Yusa et al. (2012); (3):Yamanaka et al. (2014); (4):Zheng et al. (2013); (5):Pan et al. (2015); (6):Fossey et al. (2014); (7):Zheng et al. (2014); (8):Marion et al. (2015a) ; (9):Riess et al. (2016); (10):Cortés et al. (2006) ; (11):Mazzei et al. (2017); (12):Pan et al. (2015); (13):Dale et al. (2007) 
(Johnson-Cousins/Vega and Sloan/AB for the BVRI and $g^{\prime} r^{\prime} i^{\prime} z^{\prime}$ data, respectively) was computed using catalogued Sloan-photometry for local tertiary standard stars. In the fields of SN 2012cg and SN 2012ht the $B V R I$ magnitudes for the local standards were calculated from their catalogued $g^{\prime} r^{\prime} i^{\prime} z^{\prime}$ magnitudes using the calibration given by Jordi et al. (2006). For SN 2013dy and 2014J the estimated BVRI magnitudes obtained this way were cross-checked by observing Landolt standard fields on a photometric night and re-calibrating the local standards using the zero-points from the Landolt standards. Finally, all $B V R I$ photometry were cross-compared to the Pan-STARRS (PS1) magnitudes ${ }^{2}$ of the local standards stars, and small $(\lesssim 0.1 \mathrm{mag})$ shifts were applied when needed to bring all the photometry to the same zero-point. The final $B V R I$ magnitudes for the local comparison stars are shown in the Appendix (Table 11, 13, 15 and 18).

Photometry of the SNe was obtained via PSF-fitting using DAOPHOT. The resulting instrumental magnitudes were transformed to the standard systems by applying linear color terms, and the zero points of the transformation are tied to the magnitudes of the local comparison stars.

The final LCs were compared with other published, independent photometry for each SNe, except for SN 2012ht, where no other available photometry was found. We used the data given by Marion et al. (2015b), Pan et al. (2015) and Marion et al. (2015a) for SN 2012cg, 2013dy and 2014J, respectively. $\mathrm{A} \sim 0.1 \mathrm{mag}$ systematic difference was identified between the B-band LCs of the heavily-reddened SN 2014J, which was corrected by shifting our data to match those published by Marion et al. (2015a). No such systematic offsets between our data and those from others were found for the remaining three SNe. The final photometric data can be found in the Appendix, in Tables 12, 14, 16, 17 and 19.

\section{ANALYSIS}

We applied three SN Ia LC-fitters for this study: MLCS2k $2^{3}$ (Riess et al. 1998; Jha et al. 1999; Jha, Riess \& Kirshner 2007), SALT2 $2^{4}$ version 2.4 (Guy et al. 2007, 2010; Betoule et al. 2014) and SNooPy25 (Burns et al. 2011; Burns et al. 2014). All these, in principle, rely on the Phillips-relation but each code uses different parametrization for fitting the light curve shape and each has different sets of calibrating SNe ("training sets"). There are also other implementations, like SiFTO (Conley et al. 2008) or BayeSN (Mandel et al. 2011), but the

tronomy, Inc., under cooperative agreement with the National Science Foundation.

${ }^{2}$ http://archive.stsci.edu/panstarrs/search.php

3 http: / / www.physics.rutgers.edu/ saurabh/mlcs $2 \mathrm{k} 2 /$

4 http://supernovae.in2p3.fr/salt/doku.php

5 http://csp.obs.carnegiescience.edu/data/snpy/snpy first three listed above have been used most frequently in the literature.

Conley et al. (2008) categorized such codes as either "pure LC-fitters" or "distance calculators". Distance calculators can provide the true absolute distance as a fitting parameter, but they require a training set of $\mathrm{SNe}$ having independently obtained absolute distances. Since building such a training set is non-trivial, the calibration of such codes is usually based on a relatively small number of objects. On the contrary, LC-fitters can predict only relative distances, but they can be calibrated using a much larger sample of objects having much more accurate relative distances. Regarding the three codes applied in our study, MLCS2k2 and SNooPy2 are distance calculators, while SALT2 is an LC-fitter, even though it is possible to derive absolute distances from the SALT2 fitting parameters, if needed.

MLCS2k2 (Jha, Riess \& Kirshner 2007) uses the following SN Ia LC model:

$$
m_{x}(\varphi)=M_{x}^{0}+\mu_{0}+\eta_{x} A_{V}^{0}+P_{x} \Delta+Q_{x} \Delta^{2},
$$

where $\varphi=t-T_{\max }$ is the SN phase in days, $T_{\max }$ is the moment of maximum light in the $B$-band, $m_{x}$ is the observed magnitude in the $x$-band $(x=B, V, R, I), M_{x}^{0}(\varphi)$ is the fiducial SN Ia absolute LC in the same band, $\mu_{0}$ is the true (reddening-free) SN distance modulus, $\eta_{x}=$ $\zeta_{x}\left(\alpha_{x}+\beta_{x} / R_{V}\right)$ gives the time-dependent interstellar reddening, $R_{V}$ and $A_{V}^{0}$ are the ratio of total-to-selective absorption and $V$-band extinction at maximum light, respectively, $\Delta$ is the main LC parameter, and $P_{x}(\varphi)$ and $Q_{x}(\varphi)$ are tabulated functions of the SN phase ("LC-vectors"). The absolute magnitudes of SNe in MLCS2k2 have been calibrated using relative distances of more than $150 \mathrm{SNe}$ in the Hubble-flow assuming $H_{0}=65 \mathrm{~km} \mathrm{~s}^{-1} \mathrm{Mpc}^{-1}$, but later they were tied to Cepheid distances of a smaller sample of SNe Ia host galaxies (Riess et al. 2005).

Contrary to MLCS2k2, SALT2 models the whole spectral energy distribution (SED) of a SN Ia as

$$
F_{\lambda}(\varphi)=x_{0} \cdot\left[M_{0}(\varphi, \lambda)+x_{1} M_{1}(\varphi, \lambda)\right] \exp \left[C \cdot C_{L}(\lambda)\right],
$$

where $F_{\lambda}(\varphi)$ is the phase-dependent rest-frame flux density, $M_{0}(\varphi, \lambda), M_{1}(\varphi, \lambda)$ and $C_{L}(\lambda)$ are the SALT2 trained vectors. The free parameters $x_{0}, x_{1}$ and $C$ are the normalization, stretch- and color parameters, respectively.

Being an LC-fitter, SALT2 does not contain the distance as a fitting parameter. Instead, the distance modulus can be calculated from the following equation (Conley et al. 2011; Betoule et al. 2014; Scolnic \& Kessler 2016):

$$
\mu_{0}=m_{B}^{*}-M_{B}+\alpha x_{1}-\beta C .
$$

For the nuisance parameters $\alpha, \beta$ and $M_{B}$ we adopted the calibration by Betoule et al. (2014): $M_{B}=-19.17 \pm 0.038$, 
$\alpha=0.141 \pm 0.006, \beta=3.099 \pm 0.075$, and derived the distance moduli from the fitting parameters via Monte-Carlo simulations.

One of the great advantages of SALT2 is that it can be relatively easily applied to data taken in practically any photometric system provided the filter transmission functions are loaded into the code. Because of this and many other reasons, SALT2 became very popular recently, and it was used in most papers dealing with SN Ia light curves (e.g. Betoule et al. 2014; Mosher et al. 2014; Scolnic et al. 2014; Rest et al. 2014; Saunders et al. 2015; Walker et al. 2015; Riess et al. 2016; Zhang et al. 2017). We utilized the builtin "Landolt-Bessell" and "SDSS" filter sets for fitting our $B V R I$ and $g^{\prime} r^{\prime} i^{\prime} z^{\prime}$ data, respectively.

While applying SNooPy2, we adopted the default "EBVmodel" as a proxy for a SN Ia LC:

$$
\begin{gathered}
m_{X}(\varphi)=T_{Y}+M_{Y}+\mu_{0}+K_{X Y}+ \\
R_{X} E(B-V)_{M W}+R_{Y} E(B-V)_{h o s t}
\end{gathered}
$$

where $X, Y$ denote the filter of the observed data and the template light curve, respectively, $m_{X}(\varphi)$ is the observed LC in filter $X, T_{Y}\left(\varphi, \Delta m_{15}\right)$ is the template LC as a function of time, $\Delta m_{15}$ is the generalized decline-rate parameter associated with the $\Delta m_{15}(B)$ parameter by Phillips (1993), $M_{Y}\left(\Delta m_{15}\right)$ is the absolute magnitude of the $\mathrm{SN}$ in filter $Y$ as a function of $\Delta m_{15}, \mu_{0}$ is the reddening-free distance modulus in magnitudes, $E(B-V)$ is the color excess due to interstellar extinction either in the Milky Way ("MW"), or in the host galaxy, $R_{X, Y}$ are the reddening slopes in filter $X$ or $Y$ and $K_{X Y}(t, z)$ is the cross-band $\mathrm{K}$-correction that matches the observed broad-band magnitudes of a redshifted $(z \gtrsim 0.01)$ SN taken with filter $X$ to a template SN LC in filter $Y$. Since all our SNe had very low redshift $(z<0.01)$ we always set $X=Y$ and neglected the K-corrections, which greatly simplified the analysis of those objects.

SNooPy2 offers two sets of templates which cover different filter bands. We utilized the Prieto-templates (Prieto et al. 2006) for fitting the $B V R I$ LCs, while for the $g^{\prime} r^{\prime} i^{\prime} z^{\prime}$ data we selected the built-in CSP-templates which include the $g$ , $r$ - and $i$-bands. For SN 2012cg, 2012ht and 2013dy we adopted the standard $R_{V}=3.1$ reddening slope (corresponding to the calibration=2 mode in SNooPy2), while for SN 2014J, which suffered from strong non-standard reddening, we tested both the $R_{V} \sim 1.0$ (calibration=6) and $R_{V} \sim 1.5$ (calibration=3) settings. These different calibrations are detailed in Folatelli et al. (2010).

All of these codes fit the template LCs to the observed ones via $\chi^{2}$-minimization, taking into account photometric errors as inverse weights. The optimized parameters are as follows:

- $T_{\max }$ : the moment of maximum light in the $B$-band (MJD)
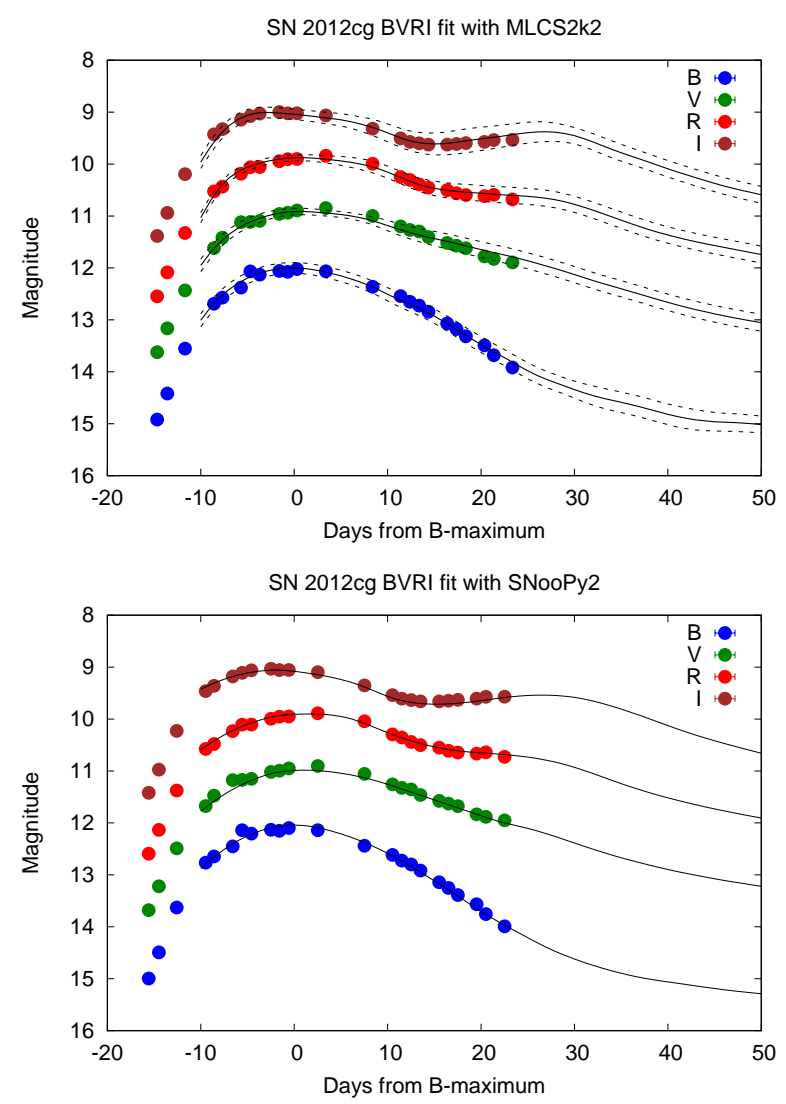

SN 2012cg BVRI fit with SALT2.4

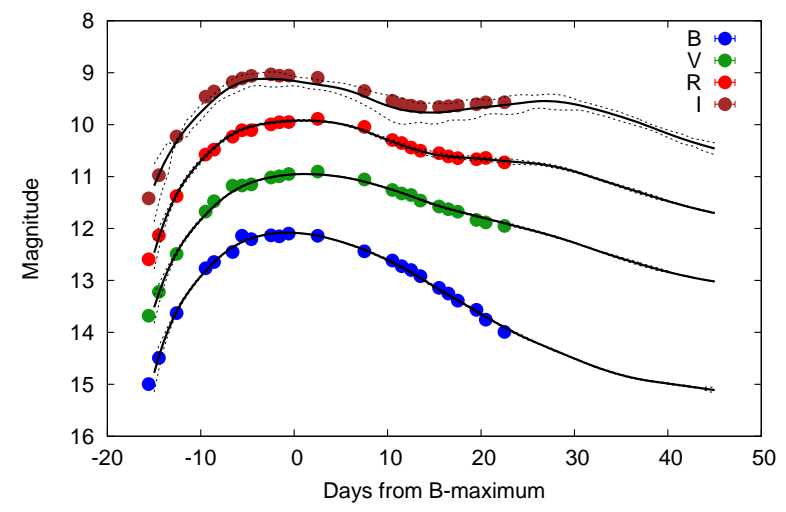

Figure 1. The fitting of SN 2012cg light curves, after correcting for Milky Way extinction. Top: MLCS2k2 templates; middle: SNooPy2 templates; bottom: SALT2.4 templates. Dashed and dotted lines represent the template uncertainties for MLCS2k2 and SALT2.4, respectively.

- $A_{V}^{\text {host }}$ : the interstellar extinction in the host galaxy in $V$-band (magnitude)

- $\mu_{0}$ : extinction-free distance modulus (magnitude)

- $\Delta$ : light curve shape parameter (MLCS2k2)

- $\Delta m_{15}$ : light curve shape parameter (SNooPy2)

- $m_{B}$ : peak brightness in $B$-band (SALT2, magnitude) 
- $x_{0}$ : light curve normalization parameter (SALT2)

- $x_{1}$ : light curve shape parameter (SALT2)

- $C$ : color parameter (SALT2)

Uncertainties of the fitting parameters were calculated via the standard analysis of the Hessian matrix of the $\chi^{2}$ hypersurface.

A particularly important goal of this study was checking the consistency of the distance moduli obtained from different photometric systems, i.e. to cross-compare the results from Johnson-Cousins $B V R I$ and Sloan $g^{\prime} r^{\prime} i^{\prime} z^{\prime}$. SALT2 and SNooPy2 are capable of handling LCs taken in $g^{\prime} r^{\prime} i^{\prime}$ bands, but not in the $z^{\prime}$-band. The publicly released version of MLCS2k2 contains only templates in $U B V R I$-bands. In order to make the analysis as complete as possible, we migrated the MLCS2k2 $U B V R I$ templates to cover the $g^{\prime} r^{\prime} i^{\prime} z^{\prime}$ filters. Details on this step are given in the Appendix.

Several studies (e.g. Hicken et al. 2009; Kelly et al. 2010; Lampeitl et al. 2010; Sullivan et al. 2010) pointed out the correlation between SN Ia peak brightnesses and host galaxy stellar masses: SNe in more massive $\left(M_{\text {stellar }} \gtrsim 10^{10} M_{\odot}\right)$ galaxies tend to be slightly brighter at peak compared to $\mathrm{SNe}$ in less massive hosts. We discuss the implication of this correlation on the derived distances in Section 4.

Previously the comparison of MLCS2k2 and SALT2 was presented by Kessler et al. (2009), who analyzed the firstseason data from the SDSS-II SN survey. They found moderate disagreement (at the $\sim 0.1-0.2 \mathrm{mag}$ level) between the distance moduli for their nearby SN sample calculated by the two codes. A similar result was obtained by Vinkó et al. (2012) when fitting the $B V R I$ data for the extremely well-observed SN $2011 \mathrm{fe}$ : the distance moduli given by MLCS2k2 and SALT2 differ by $\sim 0.16 \mathrm{mag}$. In the rest of the paper we make a similar comparison for our SN sample by involving SNooPy 2 and using data taken in different photometric systems.

We would like to emphasize that it is not intended to judge which code is superior over the others. We use these codes "as is" without any attempts to fine-tune or retrain their calibrations to get better match with any particular data, except for bringing them onto the same distance scale (see below).

\section{RESULTS}

This section summarizes the fits obtained from the different methods, and compare the results with those from previous studies, where applicable. Note that the different methods use different Hubble-parameters $\left(H_{0}\right)$ for their relative distance scales: MLCS2k2, SNooPy2 and SALT2.4 assume $H_{0}=65,72$ and $68 \mathrm{~km} \mathrm{~s}^{-1} \mathrm{Mpc}^{-1}$, respectively. In order to bring the reported distance moduli onto the same scale, all of them have been corrected to $H_{0}=73 \mathrm{~km} \mathrm{~s}^{-1} \mathrm{Mpc}^{-1}$
Table 2. Best-fit parameters for SN 2012cg

\begin{tabular}{lcc}
\hline \hline Parameter & Value & Error \\
\hline MLCS2k2 & \multicolumn{2}{c}{$B V R I$} \\
\hline$R_{V}$ & 3.1 & fixed \\
$T_{\text {max }}$ (MJD) & 56081.5 & 0.30 \\
$A_{V}^{\text {host }}$ (mag) & 0.43 & 0.05 \\
$\Delta$ (mag) & -0.19 & 0.07 \\
$\mu_{0}$ (mag) & 30.87 & 0.05 \\
$\chi^{2} /$ d.o.f. & 1.09 & \\
\hline SNooPy2 & \multicolumn{2}{c}{$B V R I$} \\
\hline$R_{V}$ & 3.1 & fixed \\
$T_{\text {max }}(\mathrm{MJD})$ & 56082.30 & 0.08 \\
$A_{V}^{\text {host }}$ (mag) & 0.46 & 0.02 \\
$\Delta m_{15}(\mathrm{mag})$ & 0.97 & 0.01 \\
$\mu_{0}$ (mag) & 30.77 & 0.02 \\
$\chi^{2} /$ d.o.f. & 0.93 & \\
\hline SALT2.4 & \multicolumn{2}{c}{$B V R I$} \\
\hline$T_{\text {max }}$ (MJD) & 56082.39 & 0.05 \\
$C$ & 0.08 & 0.02 \\
$x_{0}$ & 0.28 & 0.01 \\
$x_{1}$ & 0.45 & 0.04 \\
$m_{B}$ (mag) & 12.01 & 0.03 \\
$\mu_{0}$ (mag) & 30.85 & 0.09 \\
$\chi^{2} /$ d.o.f. & 1.44 & \\
\hline \hline & \multicolumn{2}{c}{0.09} \\
\hline
\end{tabular}

(Riess et al. 2016). The tables below contain these homogenized distance moduli. The cross-comparison of the distances obtained by these codes is further discussed in Section 5.

\section{1. $S N 2012 c g$}

Previous photometry of SN 2012cg has been presented and analyzed by Silverman et al. (2012), Munari et al. (2013), Amanullah et al. (2015) and Marion et al. (2015b). Table 2 lists the optimum parameters found by the different LC fitters applied by us. Comparing their values with the ones given by the previous studies, it is seen that they are generally consistent. There is only a slight tension between the estimated values of the extinction within the host: the results in Table 2 imply $E(B-V)_{h o s t}=A_{V}^{\text {host }} / R_{V}=0.14 \pm 0.02 \mathrm{mag}$, while Silverman et al. (2012) and Marion et al. (2015b) obtained $E(B-V)_{\text {host }} \sim 0.18 \pm 0.05$ mag which is marginally consistent with our results. Our lower reddening/extinction is closer to $E(B-V)_{\text {host }} \sim 0.13$ mag estimated by Amanullah et al. (2015). For the distance modulus, the parameter that we 
Table 3. Best-fit parameters for SN 2012ht

\begin{tabular}{|c|c|c|c|c|}
\hline Parameter & Value & Error & Value & Error \\
\hline MLCS2k2 & \multicolumn{2}{|c|}{$B V R I$} & \multicolumn{2}{|c|}{ griz } \\
\hline$R_{V}$ & 3.1 & fixed & 3.1 & fixed \\
\hline$T_{\max }(\mathrm{MJD})$ & 56295.1 & 0.30 & 56295.10 & 0.30 \\
\hline$A_{V}^{\text {host }}(\mathrm{mag})$ & 0.00 & 0.04 & 0.00 & 0.16 \\
\hline$\Delta(\mathrm{mag})$ & 0.24 & 0.04 & 0.28 & 0.12 \\
\hline$\mu_{0}$ (mag) & 32.16 & 0.04 & 32.11 & 0.14 \\
\hline$\chi^{2} /$ d.o.f. & 0.91 & & 1.73 & \\
\hline SNooPy2 & \multicolumn{2}{|c|}{$B V R I$} & \multicolumn{2}{|l|}{$g r i$} \\
\hline$R_{V}$ & 3.1 & fixed & 3.1 & fixed \\
\hline$T_{\max }(\mathrm{MJD})$ & 56295.23 & 0.08 & 56295.00 & 0.21 \\
\hline$A_{V}^{\text {host }}(\mathrm{mag})$ & 0.00 & 0.01 & 0.00 & 0.01 \\
\hline$\Delta m_{15}(\mathrm{mag})$ & 1.30 & 0.01 & 1.17 & 0.01 \\
\hline$\mu_{0}(\mathrm{mag})$ & 32.26 & 0.02 & 32.44 & 0.02 \\
\hline$\chi^{2} /$ d.o.f. & 0.58 & & 1.16 & \\
\hline SALT2.4 & \multicolumn{2}{|c|}{$B V R I$} & \multicolumn{2}{|l|}{$g r i$} \\
\hline$T_{\max }(\mathrm{MJD})$ & 56295.47 & 0.07 & 56295.50 & 0.30 \\
\hline$C$ & -0.08 & 0.03 & -0.14 & 0.05 \\
\hline$x_{0}$ & 0.111 & 0.003 & 0.116 & 0.007 \\
\hline$x_{1}$ & -1.25 & 0.05 & -0.81 & 0.26 \\
\hline$m_{B}$ (mag) & 13.02 & 0.03 & 12.98 & 0.07 \\
\hline$\mu_{0}$ (mag) & 32.12 & 0.09 & 32.32 & 0.17 \\
\hline$\chi^{2} /$ d.o.f. & 1.47 & & 2.02 & \\
\hline
\end{tabular}

focus on in this work, Munari et al. (2013) obtained $\mu_{0}=$ 30.95 assuming $E(B-V)=0.18 \mathrm{mag}$, which, after correcting to $E(B-V)=0.14 \mathrm{mag}$, corresponds to $\mu_{0}=30.83$ in very good agreement with our results presented in Table 2.

The light curves of SN 2012cg are plotted together with the models from the different LC fitters in Fig. 1.

\subsection{SN 2012ht}

A photometric study of SN 2012ht has been presented by Yamanaka et al. (2014). From their $B V R I$ photometry they have estimated the following parameters: $T_{\max }(B)=$ $56295.6 \pm 0.6, \Delta m_{15}(B)=1.39 \mathrm{mag}$ and $E(B-V)_{h o s t} \sim 0$ mag. As seen from Table 3, these are in good agreement with our results. The light curves together with the best-fit models can be found in Fig. 2. It is seen that the $B V R I$ data that have lower measurement errors could be fit better: their reduced $\chi^{2}$ values (Table 3) are lower than those of the $g^{\prime} r^{\prime} i^{\prime} z^{\prime}$ data.

In order to test the consistency of the photometric calibration of our $B V R I$ and $g^{\prime} r^{\prime} i^{\prime}$ data, simultaneous fits to
Table 4. Best-fit parameters for SN 2013dy

\begin{tabular}{|c|c|c|c|c|}
\hline Parameter & Value & Error & Value & Error \\
\hline MLCS2k2 & \multicolumn{2}{|c|}{$B V R I$} & \multicolumn{2}{|c|}{ griz } \\
\hline$R_{V}$ & 3.1 & fixed & 3.1 & fixed \\
\hline$T_{\max }(\mathrm{MJD})$ & 56500.20 & 0.30 & 56500.20 & 0.30 \\
\hline$A_{V}^{\text {host }}(\mathrm{mag})$ & 0.48 & 0.06 & 0.28 & 0.16 \\
\hline$\Delta(\mathrm{mag})$ & -0.23 & 0.06 & -0.30 & 0.12 \\
\hline$\mu_{0}(\mathrm{mag})$ & 31.51 & 0.06 & 31.65 & 0.13 \\
\hline$\chi^{2} /$ d.o.f. & 1.34 & & 1.91 & \\
\hline SNooPy2 & \multicolumn{2}{|c|}{$B V R I$} & \multicolumn{2}{|c|}{$g r i$} \\
\hline$R_{V}$ & 3.1 & fixed & 3.1 & fixed \\
\hline$T_{\max }(\mathrm{MJD})$ & 56501.30 & 0.08 & 56501.03 & 0.15 \\
\hline$A_{V}^{\text {host }}(\mathrm{mag})$ & 0.40 & 0.02 & 0.61 & 0.05 \\
\hline$\Delta m_{15}(\mathrm{mag})$ & 0.96 & 0.01 & 0.80 & 0.01 \\
\hline$\mu_{0}(\mathrm{mag})$ & 31.52 & 0.03 & 31.44 & 0.04 \\
\hline$\chi^{2} /$ d.o.f. & 0.99 & & 3.45 & \\
\hline SALT2.4 & \multicolumn{2}{|c|}{$B V R I$} & \multicolumn{2}{|c|}{$g r i$} \\
\hline$T_{\max }(\mathrm{MJD})$ & 56501.44 & 0.06 & 56501.99 & 0.14 \\
\hline$C$ & 0.089 & 0.025 & 0.03 & 0.03 \\
\hline$x_{0}$ & 0.154 & 0.004 & 0.169 & 0.006 \\
\hline$x_{1}$ & 0.695 & 0.044 & 1.51 & 0.12 \\
\hline$m_{B}(\mathrm{mag})$ & 12.67 & 0.03 & 12.57 & 0.04 \\
\hline$\mu_{0}(\mathrm{mag})$ & 31.52 & 0.09 & 31.72 & 0.11 \\
\hline$\chi^{2} /$ d.o.f. & 0.76 & & 2.70 & \\
\hline
\end{tabular}

the combined LCs were also computed with SNooPy2 and SALT2.4 (MLCS2k2 was trained only on $B V R I$ data, so that code was not applied in this test). As expected, these fits produced slightly higher $\chi^{2}$ values than the fits to the $B V R I$ LCs alone, but their best-fit parameters were consistent with the ones listed in Table 3. In particular, the distance modulus from the combined fits turned out to be $\mu_{0}=32.20 \pm 0.01$ mag $\left(\chi^{2} /\right.$ d.o.f $\left.=0.83\right)$ from SNooPy2, while from SALT2.4 it is $32.13 \pm 0.08 \mathrm{mag}\left(\chi^{2} /\right.$ d.o.f.=2.81). These parameters are closer to those obtained from fitting the $B V R I$ LCs than those from fitting the $g^{\prime} r^{\prime} i^{\prime} z^{\prime}$ data, probably because of the lower measurement uncertainties of the former.

\subsection{SN 2013dy}

Light curves of SN 2013dy have been published recently by Pan et al. (2015) (P15) and Zhai et al. (2016) (Z16) in the $B V R I r i Z Y J H$ and $U B V R I$ bands, respectively. Zhai et al. (2016) also presented photometry obtained by Swift/UVOT. 

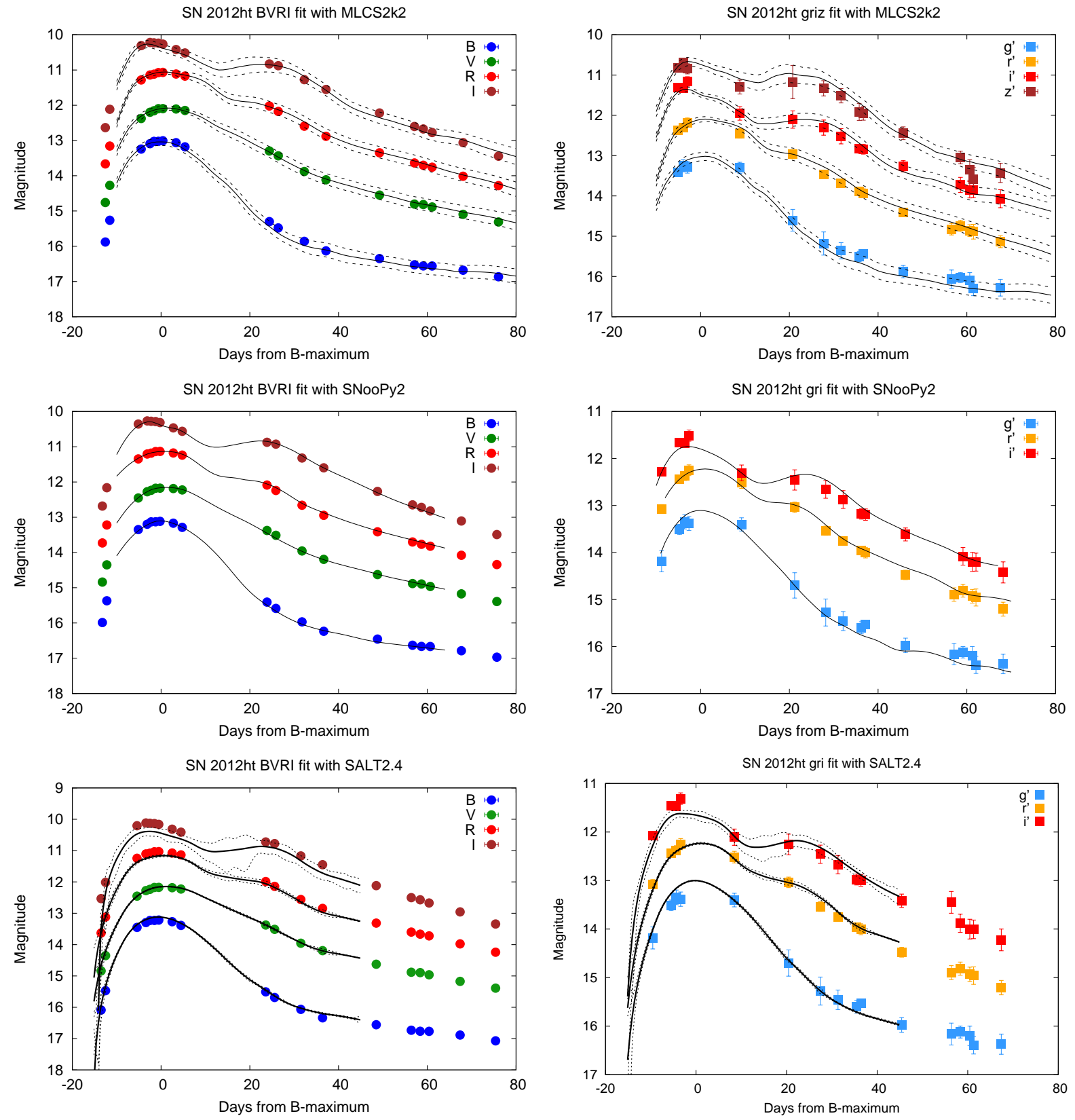

Figure 2. The fitting of the light curves of SN 2012ht, after correcting for Milky Way extinction. Top row: MLCS2k2; middle row: SNooPy2; bottom row: SALT2.4; left column: $B V R I$ data; right column: $g^{\prime} r^{\prime} i^{\prime} z^{\prime}$ data.

Pan et al. (2015) applied the SNooPy2 code to fit their full BVRIriZYJH dataset simultaneously, and obtained $T_{\max }(B)=56501.1, \Delta m_{15}=0.886 \pm 0.006, E(B-$ $V)_{\text {host }}=0.206 \pm 0.005 \mathrm{mag}$ and $\mu_{0}=31.49 \pm 0.01 \mathrm{mag}$. Comparing these values with those in Table 4 it is apparent that the results of Pan et al. (2015) are close to the ones obtained in the present study, although the differences somewhat exceed the formal errors given by SNooPy2. Comparing the best-fit values of the common parameters obtained from different methods, e.g. $A_{V}^{\text {host }}$ or $\mu_{0}$, it is seen that they also deviate much more than the uncertainties given by the codes. Thus, it is suspected that the formal parameter errors, especially those reported by SNooPy2, are underestimated, and the true uncertainties should be higher. Keeping this in mind, the solutions presented in Table 4 are entirely consistent with the LC fit given by Pan et al. (2015). The fit of the model LCs to the data can be seen in Fig. 3.

We re-analyzed the $B V R I$ light curves of SN 2013dy from both Pan et al. (2015) and Zhai et al. (2016) with all three methods applied in this paper in order to cross-compare the results from fitting measurements taken independently on the same SN. We assumed $R_{V}=3.1$ for all fits, as earlier. The 

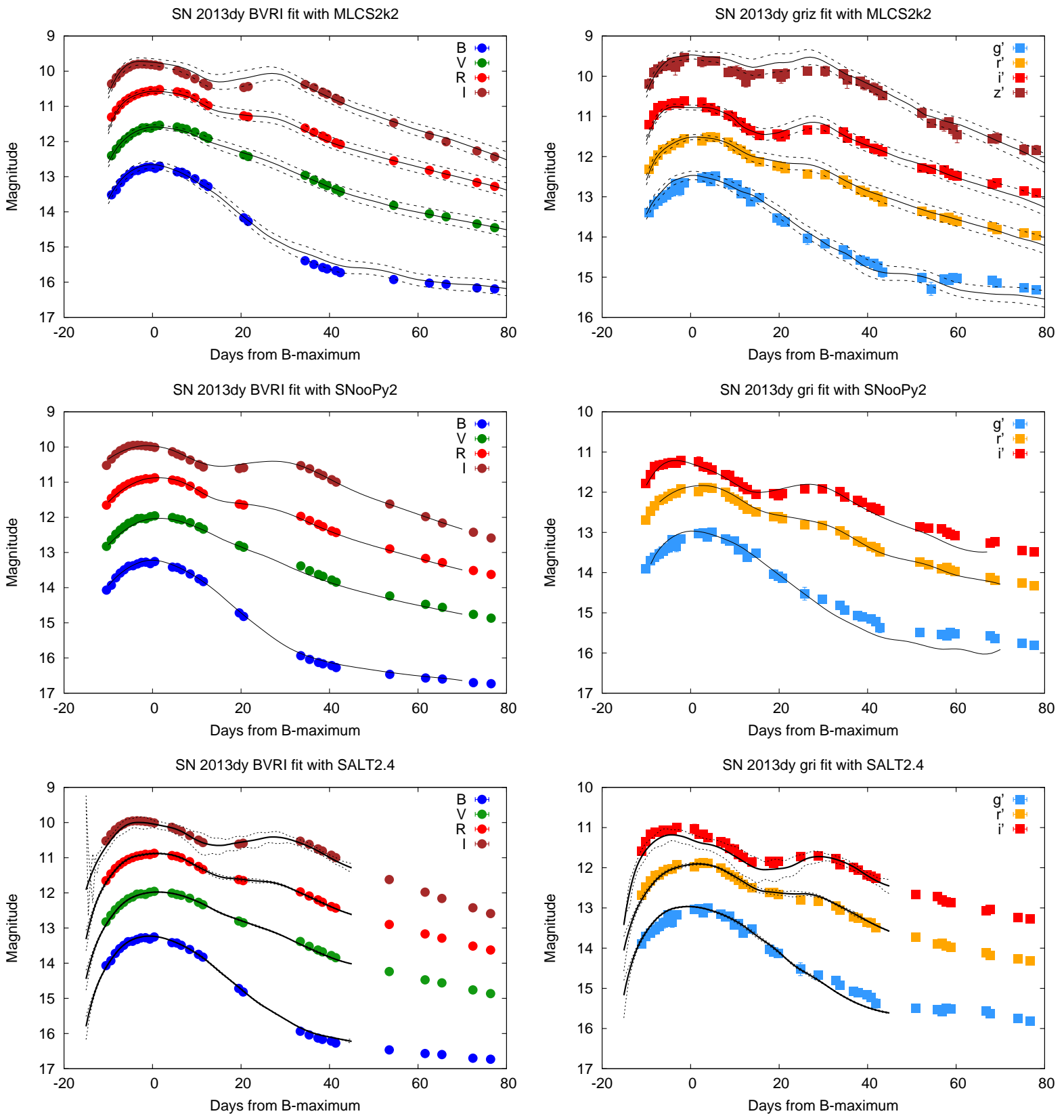

Figure 3. The same as Fig.2 but for SN 2013dy.

results are shown in Table 5. It is seen that the consistency between the distance moduli from the three methods is excellent for all data. Overall, the distance moduli from the Konkoly data differ by less than $\sim 1 \sigma$ ( $\lesssim 0.1 \mathrm{mag}$ ) from both the P15 and Z16 results. Also, it is seen from Table 5 that similar amount of dispersion in the distance moduli derived by the different codes from the same dataset appears for the P15 and Z16 LCs as well as for our data, which suggests that this dispersion is probably not simply due to photometric calibration issues, at least for the $B V R I$ data.

In addition, we also modeled the combined $B V R I+g^{\prime} r^{\prime} i$ LCs as in the case for SN 2012ht. The resulting distance moduli are $\mu_{0}=31.45 \pm 0.01 \mathrm{mag}\left(\chi^{2} /\right.$ d.o.f.=2.92) from SNooPy 2 and $\mu_{0}=31.54 \pm 0.07 \mathrm{mag}\left(\chi^{2} /\right.$ d.o.f. $\left.=2.11\right)$ from SALT2.4. Again, these results are in good agreement with those listed in Table 4, even though the $\chi^{2}$ values of the combined fits are somewhat higher but still acceptable (note again that the uncertainty of $\mu_{0}$ reported by SNooPy 2 is underestimated).

\subsection{SN 2014J}

As seen in Table 6, the LCs of SN 2014J have been fit with two different models assuming different reddening laws for the host galaxy in MLCS2k2 and SNooPy2. This was 

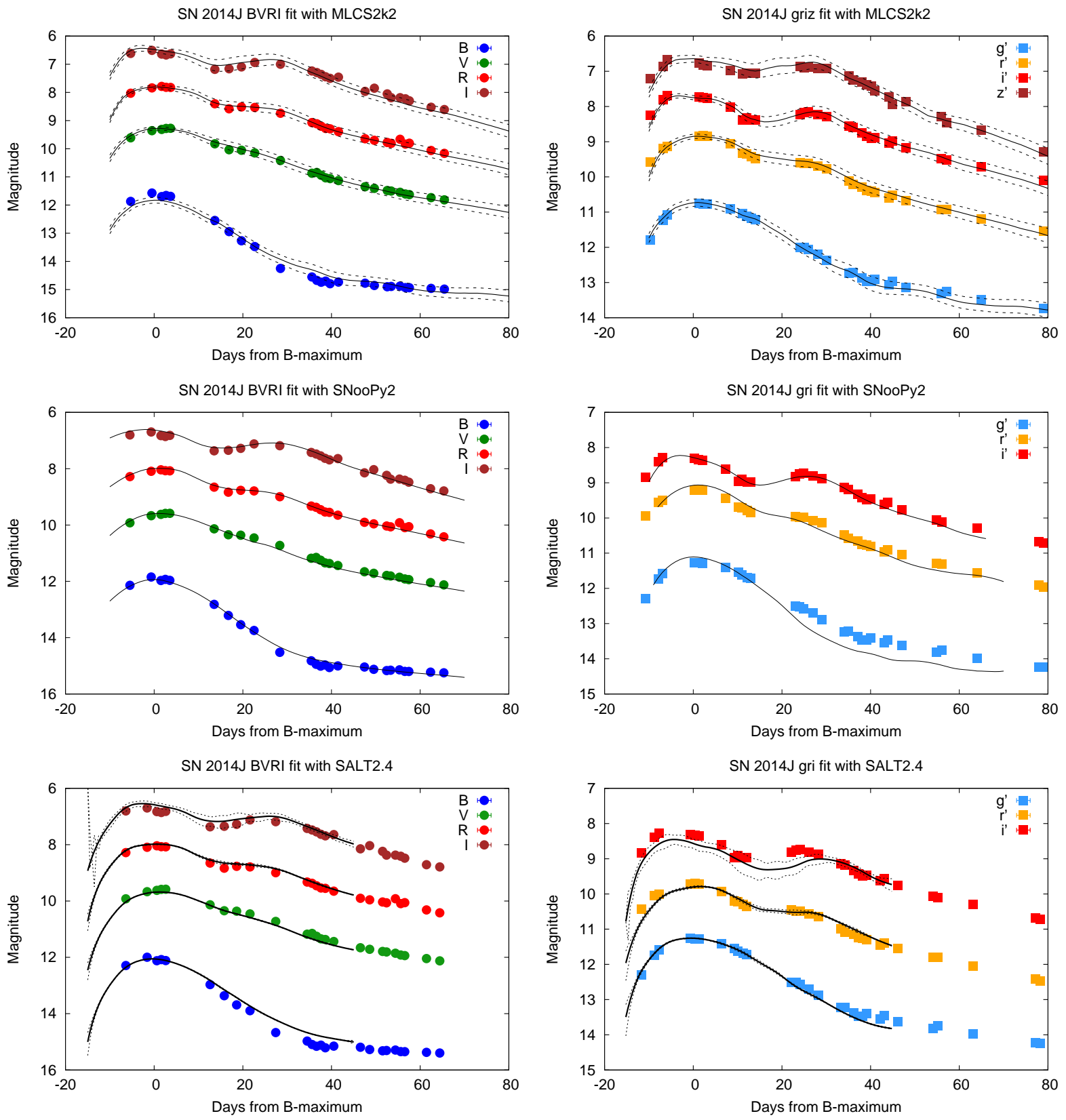

Figure 4. The same as Fig.2 but for SN 2014J.

motivated by the fact that many studies (see below) found $R_{V}<2$ in M82, quite different from the Milky Way value of $R_{V}=3.1$.

When using MLCS2k2, we considered two different scenarios: first, we adopted $A_{V}^{M W}=0.43 \mathrm{mag}$ from the extinction map of Schlafly \& Finkbeiner (2011) at the position of SN 2014J, and $R_{V}=1.4$ based on the results of Goobar et al. (2014), Foley et al. (2014) and Amanullah et al. (2014). Secondly, we let $R_{V}$ float until the lowest $\chi^{2}$ was found by MLCS2k2. This resulted in $R_{V} \sim 1.0$, and the parameters corresponding to this solution are adopted as the best-fit MLCS2k2 values (see Table 6).
Note that such a low value of $R_{V}$ is close to the limiting case of Rayleigh scattering from very small particles, producing $R_{V} \sim 1.2$ (Draine 2003). From the full sample of the SDSSII SN survey (361 SNe Ia) Lampeitl et al. (2010) found that the average extinction law for $\mathrm{SNe}$ in passive host galaxies is $R_{V}=1.0 \pm 0.2$. Thus, even though the host of SN 2014J, M82, is an extremely active star-forming galaxy, such a low value for the extinction law is not unprecedented.

In SNooPy2, different reddening laws are implemented as different "calibrations" (Burns et al. 2014). We applied both the calibration=3 and calibration=6 settings (cor- 
Table 5. Cross-comparison of the parameters from fitting independent data on SN 2013dy. Uncertainties are in parentheses.

\begin{tabular}{llll}
\hline \hline Parameter & this work & $\mathrm{P} 15$ & $\mathrm{Z} 16$ \\
\hline MLCS2k2: & & & \\
$T_{\max }$ & $56500.2(0.3)$ & $56500.5(0.3)$ & $56501.4(0.3)$ \\
$A_{V}^{\text {host }}$ & $0.48(0.06)$ & $0.42(0.07)$ & $0.45(0.06)$ \\
$\Delta$ & $-0.23(0.06)$ & $-0.20(0.05)$ & $-0.22(0.05)$ \\
$\mu_{0}$ & $31.51(0.06)$ & $31.53(0.06)$ & $31.53(0.07)$ \\
\hline SNooPy2: & & & \\
$T_{\text {max }}$ & $56501.30(0.08)$ & $56501.48(0.11)$ & $56501.29(0.11)$ \\
$A_{V}^{\text {host }}$ & $0.40(0.02)$ & $0.31(0.02)$ & $0.43(0.02)$ \\
$\Delta m_{15}$ & $0.96(0.01)$ & $0.97(0.02)$ & $0.86(0.02)$ \\
$\mu_{0}$ & $31.52(0.03)$ & $31.56(0.01)$ & $31.52(0.02)$ \\
\hline SALT2.4: & & & \\
$T_{\max }$ & $56501.44(0.06)$ & $56501.47(0.04)$ & $56502.09(0.14)$ \\
$C$ & $0.089(0.025)$ & $0.081(0.016)$ & $0.149(0.024)$ \\
$x_{0}$ & $0.154(0.004)$ & $0.152(0.003)$ & $0.143(0.004)$ \\
$x_{1}$ & $0.695(0.044)$ & $0.814(0.044)$ & $1.002(0.073)$ \\
$m_{B}$ & $12.670(0.028)$ & $12.682(0.025)$ & $12.743(0.028)$ \\
$\mu_{0}$ & $31.52(0.08)$ & $31.573(0.069)$ & $31.450(0.090)$ \\
\hline \hline & & & \\
\hline
\end{tabular}

responding to $R_{V} \sim 1.5$ and $R_{V} \sim 1.0$, respectively), and list the best-fit parameters for each in Table 6 .

Note that Marion et al. (2015a) adopted a different Milky Way extinction value toward SN 2014J (they used $E(B-$ $V)_{M W}=0.05 \mathrm{mag}$ corresponding to $A_{V}^{M W}=0.16 \mathrm{mag}$ ), because the dust content of M82 may influence the far-IR maps of Schlafly \& Finkbeiner (2011) in that direction. Using this lower Milky Way extinction parameter one would get $\sim 0.1$ mag higher $A_{V}^{\text {host }}$ and $\sim 0.15$ mag higher distance modulus for SN 2014J. While keeping this in mind, in the following we use the higher Milky Way extinction value as given by the reddening maps of Schlafly \& Finkbeiner (2011). In this case the MLCS2k2 results are directly comparable to the ones derived by SNooPy2, because SNooPy2 automatically applies the Schlafly \& Finkbeiner (2011) values for calculating the Milky Way extinction.

SALT2.4 fits the reddening of the SN in a different way: instead of applying the same dust extinction law as MLCS2k2 or SNooPy2, it models the reddening via the $C$ color parameter (see Eq.2). Thus, the effect of the strong interstellar extinction on the LCs of SN 2014J is reflected by the extremely large value of its SALT2.4 color coefficient, which is more than an order of magnitude higher than for the other three SNe.

The light curves are plotted in Fig. 4.
Table 6. Best-fit parameters for SN 2014J

\begin{tabular}{|c|c|c|c|c|}
\hline Parameter & Value & Error & Value & Error \\
\hline MLCS2k2 & \multicolumn{2}{|c|}{$B V R I$} & \multicolumn{2}{|c|}{ griz } \\
\hline$R_{V}$ & 1.4 & fixed & 1.4 & fixed \\
\hline$T_{\max }(\mathrm{MJD})$ & 56689.8 & 0.50 & 56689.8 & 0.50 \\
\hline$A_{V}^{\text {host }}$ (mag) & 1.84 & 0.09 & 1.49 & 0.16 \\
\hline$\Delta(\operatorname{mag})$ & -0.13 & 0.08 & -0.24 & 0.12 \\
\hline$\mu_{0}(\mathrm{mag})$ & 27.72 & 0.09 & 27.99 & 0.13 \\
\hline$\chi^{2} /$ d.o.f. & 3.62 & & 2.37 & \\
\hline$R_{V}$ & 1.0 & fixed & 1.0 & fixed \\
\hline$T_{\max }(\mathrm{MJD})$ & 56689.8 & 0.50 & 56689.8 & 0.50 \\
\hline$A_{V}^{\text {host }}$ (mag) & 1.41 & 0.08 & 1.16 & 0.16 \\
\hline$\Delta(\mathrm{mag})$ & -0.16 & 0.07 & -0.25 & 0.12 \\
\hline$\mu_{0}(\mathrm{mag})$ & 28.14 & 0.08 & 28.21 & 0.13 \\
\hline$\chi^{2} /$ d.o.f. & 3.15 & & 2.32 & \\
\hline SNooPy2 & \multicolumn{2}{|c|}{$B V R I$} & \multicolumn{2}{|l|}{$g r i$} \\
\hline$R_{V}$ & 1.5 & fixed & 1.5 & fixed \\
\hline$T_{\max }(\mathrm{MJD})$ & 56689.99 & 0.42 & 56690.86 & 0.24 \\
\hline$A_{V}^{\text {host }}$ (mag) & 1.89 & 0.05 & 1.40 & 0.03 \\
\hline$\Delta m_{15}(\mathrm{mag})$ & 1.04 & 0.04 & 1.05 & 0.03 \\
\hline$\mu_{0}(\mathrm{mag})$ & 27.50 & 0.02 & 27.69 & 0.05 \\
\hline$\chi^{2} /$ d.o.f. & 4.21 & & 16.73 & \\
\hline$R_{V}$ & 1.0 & fixed & 1.0 & fixed \\
\hline$T_{\max }(\mathrm{MJD})$ & 56689.99 & 0.34 & 56690.86 & 0.24 \\
\hline$A_{V}^{\text {host }}(\mathrm{mag})$ & 1.34 & 0.03 & 0.98 & 0.02 \\
\hline$\Delta m_{15}(\mathrm{mag})$ & 1.04 & 0.04 & 1.06 & 0.03 \\
\hline$\mu_{0}(\mathrm{mag})$ & 27.99 & 0.02 & 28.01 & 0.05 \\
\hline$\chi^{2} /$ d.o.f. & 2.99 & & 17.13 & \\
\hline SALT2.4 & \multicolumn{2}{|c|}{$B V R I$} & \multicolumn{2}{|c|}{$g r i$} \\
\hline$T_{\max }(\mathrm{MJD})$ & 56690.88 & 0.11 & 56691.49 & 0.11 \\
\hline$C$ & 1.22 & 0.03 & 0.86 & 0.03 \\
\hline$x_{0}$ & 0.416 & 0.013 & 0.544 & 0.017 \\
\hline$x_{1}$ & 0.03 & 0.06 & 1.39 & 0.07 \\
\hline$m_{B}(\mathrm{mag})$ & 11.50 & 0.03 & 11.25 & 0.03 \\
\hline$\mu_{0}(\mathrm{mag})$ & 26.74 & 0.13 & 27.80 & 0.12 \\
\hline$\chi^{2} /$ d.o.f. & 5.83 & & 5.87 & \\
\hline
\end{tabular}

Applying SNooPy2 on their own $U B V R I J H K$ photometry, Marion et al. (2015a) obtained $T_{\max }(B)=56689.74 \pm$ $0.13 \mathrm{MJD}, d m_{15}(B)=1.11 \pm 0.02, E(B-V)_{h o s t}=1.23 \pm$ 0.01 and $\mu_{0}=27.85 \pm 0.09$ mag in calibration $=4$ mode $\left(R_{V} \sim 1.46\right)$. Their reddening value, $E(B-V)_{h o s t}$, implies 
$A_{V}^{\text {host }}=1.80 \pm 0.03 \mathrm{mag}$. These parameters are marginally consistent with our results listed in Table 6. However, the relatively large differences between the distance moduli obtained from the $B V R I$ and griz data, and also between the results from MLCS2k2 and SNooPy2, suggest that the $R_{V} \sim 1.4$ solution may not be the best one as far as LC fitting is concerned. Indeed, by comparing the distance moduli obtained from the $R_{V} \sim 1.0$ solutions, it seems that those values are more consistent with each other. The average $\mu_{0}$ for the latter solution is $\sim 28.09 \pm 0.11 \mathrm{mag}$, while it is $\sim 27.72 \pm 0.20$ mag for the $R_{V} \sim 1.4$ solution. The dispersion of the distance moduli derived from the two sets of light curves and two independent codes is much less when $R_{V}=1.0$ is used for the M82 reddening law, compared to the $R_{V} \sim 1.4$ case adopted by Marion et al. (2015a).

Fitting the combined $B V R I+g^{\prime} r^{\prime} i^{\prime}$ dataset with SNooPy2 gave distance moduli similar to those listed in Table 6: $\mu_{0}=$ $27.50 \pm 0.02$ (calibration $=3$ ) and $\mu_{0}=28.02 \pm 0.02$ (calibration $=6$ ). However, the reduced $\chi^{2}$ values for the combined fits were 31.66 and 34.79 , respectively, indicating poor fitting quality. Comparing the best-fit template LCs with the observed ones revealed that the $g^{\prime}$ band data could not be fit simultaneously with the other bands: while the shape of the template LC was similar to the observed one, the observed $g^{\prime}$-band LC was too bright (by $\sim 0.5 \mathrm{mag}$ ) with respect to the template. This could be due to either an issue with our photometry (which is unlikely given that the other data do not show such a high deviation), or the complexity of the reddening law in M82 that may not be fully modeled by a single $R_{V}$ (Foley et al. 2014).

The SALT2.4 code could not provide reliable distances for this heavily reddened SN. The SALT2.4 distances for SN 2014J are inconsistent with each other, as well as with the distances given by the other two codes. SALT2.4 also failed to give consistent fits to the combined $B V R I+g^{\prime} r^{\prime} i^{\prime}$ LCs: neither of the templates matched the observed data adequately, resulting in $\chi^{2}>50$.

We conclude that for SN 2014J only MLCS2k2 and SNooPy2 were able to provide more-or-less consistent distances, and both of those LC fitters suggest $R_{V} \sim 1.0$, i.e. a lower value than found by the spectroscopic studies. However, the failure of the simultaneous fitting of the combined LCs suggest that the complexity of the extinction within M82 may affect the derived distances to SN 2014J more than in the other three cases.

\subsection{Correction for the host galaxy mass}

The distance moduli given in Tables 2-6 do not contain the correction for the host galaxy mass (see Section 1 for references). Betoule et al. (2014) found that SNe Ia that exploded in host galaxies having total stellar mass of $M_{\text {stellar }}>10^{10}$ $M_{\odot}$ are $\sim 0.06$ mag brighter than those in less massive hosts.
The calibration of SALT2.4 by Betoule et al. (2014) that we applied in this paper already contains this so-called "massstep": the $M_{B}$ parameter given after Eq.3 is valid for $\mathrm{SNe}$ in less massive hosts, and it is $M_{B}-0.061 \mathrm{mag}$ for $\mathrm{SNe}$ in more massive hosts.

The stellar masses for the host galaxies in this paper are listed in Table 1. These were derived, following Pan et al. (2014), by applying Z-PEG ${ }^{6}$ (Le Borgne \& Rocca-Volmerange 2002) to the observed galaxy SEDs (see Table 1 for references). It is seen that only SN $2014 \mathrm{~J}$ is affected by this correction, since the hosts of the other three $\mathrm{SNe}$ are below $\log M_{\text {stellar }}=10$. Thus, their $M_{B}$ parameter does not need to be corrected, and their SALT2.4 distances are final. Unfortunately, the SALT2.4 distance moduli of SN 2014J are unreliable as they are affected by the strong non-standard interstellar extinction (see the previous subsection). These systematic uncertainties ( $\gtrsim 0.5 \mathrm{mag}$; Table 6$)$ are much higher than the correction for the host galaxy mass $(\sim 0.06$ mag) in the case of SN 2014J.

Nevertheless, we investigated whether the mass-step correction could bring the derived distances to better agreement with each other for the other three SNe. Since neither MLCS2k2, nor SNooPy2 contain the mass-step correction in their calibrations, we followed the practice applied by Riess et al. (2016) by adding 0.03 mag to the MLCS2k2/SNooPy2 distance moduli of SN 2014J and subtracting 0.03 mag from the distances of SNe 2012cg, 2012ht and $2013 \mathrm{dy}$, thus, mimicking the existence of the mass-step in their peak brightnesses.

Table 7 shows the differences between the distance moduli estimated by SALT2.4 and the other two codes for both photometric systems after implementing the host mass correction as described above. For comparison, we give the same differences between the uncorrected distances in parentheses. Ideally, after correction all these differences should be zero. In reality, it is apparent that the effect of the host mass correction is minimal: sometimes it makes the agreement slightly better, sometimes slightly worse, but its amount $( \pm 0.03 \mathrm{mag})$ is an order of magnitude less than the differences between the distance moduli given by the different LC-fitting codes.

It is concluded that the host mass correction on the distance modulus is negligible compared to the other sources of uncertainty, at least for the SNe studied in this paper. Note, however, that in studies using relative distances, such as Betoule et al. (2014); Riess et al. (2016) and others, this effect can be much more important and significant. Therefore, in the rest of the paper we use the uncorrected distances (i.e. without the mass-step) as given in Tables 2-6, but note

\footnotetext{
${ }^{6}$ http://imacdlb.iap.fr/cgi-bin/zpeg/zpeg.pl
} 
Table 7. Differences in distance moduli after corrections for host galaxy masses

\begin{tabular}{lcccc}
\hline \hline SN & SALT-MLCS & SALT-MLCS & SALT-SNooPy & SALT-SNooPy \\
& $B V R I(\mathrm{mag})$ & $g^{\prime} r^{\prime} i^{\prime} z^{\prime}(\mathrm{mag})$ & $B V R I(\mathrm{mag})$ & $g^{\prime} r^{\prime} i^{\prime}(\mathrm{mag})$ \\
\hline $2012 \mathrm{cg}$ & $0.01(-0.02)$ & - & $0.11(0.08)$ & - \\
$2012 \mathrm{ht}$ & $-0.01(-0.04)$ & $0.24(0.21)$ & $-0.11(-0.14)$ & $-0.09(-0.12)$ \\
$2013 \mathrm{dy}$ & $0.04(0.01)$ & $0.1(0.07)$ & $0.03(0.00)$ & $0.31(0.28)$ \\
\hline \hline
\end{tabular}

NOTE-Differences between the uncorrected distance moduli are given in parentheses.

that it is desirable to include the host mass correction in future retraining of the MLCS2k2 and/or SNooPy2 templates.

\section{DISCUSSION}

In this section we cross-compare the parameters derived by the three independent codes, and check the consistency between the values inferred from different photometric systems ( $B V R I$ vs griz) and by different LC fitters.

\subsection{Time of maximum light}

For Type Ia SNe the moment of maximum light in the $B$ band has been used traditionally as the zero-point of time. At first it seems to be fairly easy to measure directly from the data, at least when the LC in $B$-band is available. From Tables $2-6$ it is seen that the LC fitters used in this study do a good job in estimating $T_{\max }(B)$ even if the $B$-band LC is not included in the fitting. The consistency between the derived values is also relatively good: the dispersion around the mean values is $0.49,0.21,0.71$ and 0.67 day for SN $2012 \mathrm{cg}$, 2012ht, 2013dy and 2014J, respectively. Note, however, that SALT2.4 gets later maximum times systematically by $\Delta t>0.5$ day relative to MLCS2k2, similar to the finding by Vinkó et al. (2012) and Pereira et al. (2013) for SN 2011fe.

\subsection{Extinction}

The host galaxy dust extinction parameters $\left(A_{V}^{\text {host }}\right)$ in Tables 2 - 6 look generally consistent with each other. The match between the values provided by the same code for $B V R I$ and griz is usually better than the agreement between the results of the different codes (here only MLCS2k2 and SNooPy2 are relevant, because SALT2.4 does not model dust extinction).

As an independent sanity check, we compare the average $A_{V}^{\text {host }}$ values from MLCS2k2 and SNooPy2 for SN 2012ht and 2013dy with high-resolution spectra obtained with the Hobby-Eberly Telescope (HET). For SN 2013dy these spectra were published by Pan et al. (2015), while those for SN 2012ht are yet unpublished. In Fig. 5 the spectral regions containing the $\mathrm{Na} \mathrm{D}(\lambda \lambda 5890,5896)$ doublet are shown. The narrow $\mathrm{Na} \mathrm{D}$ features originating from the ISM both in the Milky Way and in the host galaxy (separated by the Dopplershift due to the recession velocity of the host) are labeled.
The strength of the Na D doublet is thought to be proportional to the amount of extinction, at least as a first approximation (Richmond et al. 1994; Poznanski et al. 2012). It is seen that the dust extinction within the host galaxy for SN 2012ht is negligible (no narrow $\mathrm{Na} \mathrm{D}$ absorption is visible at the redshift of the host) compared to the Milky Way component. This is in excellent agreement with the predictions from the LC fitters, because both codes resulted in $A_{V}^{\text {host }}=0$ magnitude for SN 2012ht.

Concerning SN 2013dy, the consistency between the photometric and spectroscopic extinction estimates is also very good. In Fig. 5 the $\mathrm{Na} \mathrm{D}$ profiles in the host galaxy have approximately the same strength as the Milky Way components. From Tables 1 and $4, A_{V}^{M W}=0.42 \mathrm{mag}$ and $A_{V}^{\text {host }} \sim 0.44 \pm 0.14$ mag were taken for SN $2013 \mathrm{dy}$, which are, again, in good agreement with the relative strengths of the $\mathrm{Na} \mathrm{D}$ profiles in the high-resolution spectra.

The case of SN 2014J is more problematic, as this SN occured within a host galaxy that has a known complex dust content. Foley et al. (2014) presented an in-depth study of the wavelength-dependent reddening and extinction toward SN 2014J, and concluded that it is probably much more complex than a simple extinction law parametrized by a single value of $A_{V}$ and $R_{V}$. Keeping this in mind, it is not surprising that the LC-fitting codes applied in this paper failed to produce consistent results with the spectroscopic estimates (Amanullah et al. 2014; Foley et al. 2014; Goobar et al. 2014; Brown et al. 2015; Gao et al. 2015) that all found $R_{V} \gtrsim 1.4$.

\subsection{Light curve shape/stretch parameter}

The light curve shape/stretch parameter is the one that is most strongly connected to the peak brightness of a Type Ia SN; thus, it has a direct influence on the distance measurement. Since the three LC-fitting codes adopt slightly different parametrizations of the light curve shape, we converted each of them to the traditional $\Delta m_{15}(B)$ (Phillips 1993). From the MLCS2k2 templates we get $\Delta m_{15}(B)=1.07+0.67 \cdot \Delta-0.10 \cdot \Delta^{2}$. For SNooPy 2 we adopted $\Delta m_{15}(B)=0.13+0.89 \cdot \Delta m_{15}$ (Burns et al. 2011), while for SALT2.4 we used $\Delta m_{15}(B)=1.09-$ $0.161 \cdot x_{1}+0.013 \cdot x_{1}^{2}-0.0013 \cdot x_{1}^{3}$ (Guy et al. 2007). 

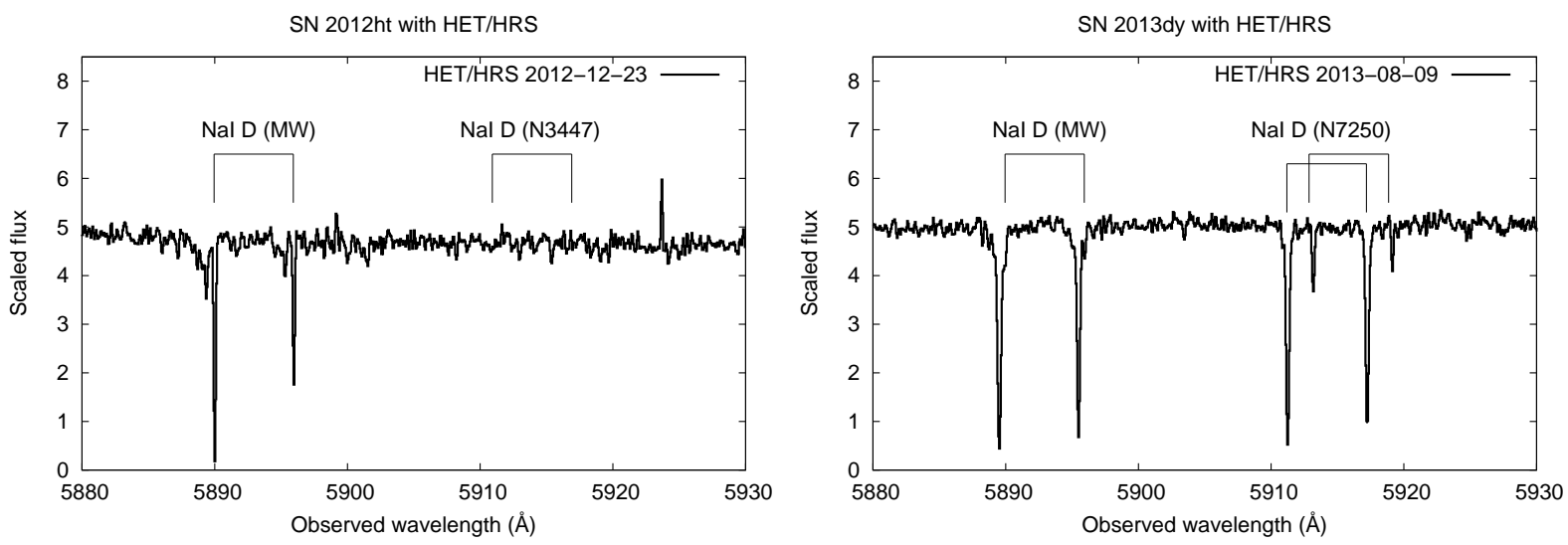

Figure 5. The narrow NaD features in the high-resolution spectrum of SN 2012ht taken at 11 days before maximum (left panel) and 2013dy at 12 days after maximum (right panel). The components from the Milky Way and host galaxy are labeled. See text for details.

Table 8.

\begin{tabular}{lccc}
\hline \hline SN & $\Delta m_{15}(B)$ & $\Delta m_{15}(B)$ & $\Delta m_{15}(B)$ \\
& $B V R I(\mathrm{mag})$ & $g^{\prime} r^{\prime} i^{\prime} z^{\prime}(\mathrm{mag})$ & combined (mag) \\
\hline $2012 \mathrm{cg}$ & $0.984(0.041)$ & - & - \\
$2012 \mathrm{ht}$ & $1.275(0.045)$ & $1.217(0.041)$ & $1.298(0.010)$ \\
$2013 \mathrm{dy}$ & $0.960(0.043)$ & $0.858(0.015)$ & $0.971(0.007)$ \\
2014J & $1.034(0.065)$ & $0.952(0.105)$ & $0.981(0.074)$ \\
\hline \hline
\end{tabular}

NotE-Standard deviations are given in parentheses.

Table 8 lists the resulting $\Delta m_{15}(B)$ values, averaged over the three methods, for the $B V R I$ and $g^{\prime} r^{\prime} i^{\prime} z^{\prime}$ data and for the combined fits, respectively. It is seen that the fits to the $g^{\prime} r^{\prime} i^{\prime} z^{\prime}$ data alone tend to result in systematically lower decline rates than the fits to the $B V R I$ data or the combined $B V R I+g^{\prime} r^{\prime} i^{\prime}$ data. The deviation is the highest in the case of SN 2013dy, $0.1 \mathrm{mag}$, and it is lower for the other two SNe. Neglecting the differences between the other fitting parameters, an underestimate of the decline rate by $\sim 0.1$ mag would cause an overestimate of $\sim 0.08 \mathrm{mag}$ in the distance modulus (applying the decline rate - absolute peak magnitude calibration by Burns et al. (2014)). Since the derived distance moduli do not show such a systematic trend between the $B V R I$ and $g^{\prime} r^{\prime} i^{\prime} z^{\prime}$ data, and the differences between them are sometimes higher than $0.08 \mathrm{mag}$ (see next section), it is concluded that the systematic underestimate of the decline rates from our $g^{\prime} r^{\prime} i^{\prime} z^{\prime}$ photometry is not significant, at least from the present dataset. The number of $\mathrm{SNe}$ in this paper is too low to draw more definite conclusions on the cause of the dependence of the light curve shape/stretch parameter on the photometric bands (whether it is merely due to uncertainties in the data or might have physical origin), but it would be worth studying on a larger sample of SNe.

\subsection{Distance}

Distance is one of the most important outputs of the LC fitting codes under study. Cross-comparing the distance estimates produced by the various codes may reveal important constraints on the systematics that are present either in the basic assumptions of the methods or in their implementation and calibrations.

For the extremely well-observed SN $2011 \mathrm{fe}$, Vinkó et al. (2012) found a $0.16 \pm 0.07$ mag systematic difference $(\sim 2 \sigma)$ between the distance moduli provided by MLCS2k2 and SALT2, when applied for the same homogeneous photometric data. In the left panel of Fig. 6 we plot the residual distance moduli (i.e. the difference of the distance modulus given by the LC-fitting code for each filter set, $B V R I$ or griz, from the mean distance modulus) for each $\mathrm{SNe}$ as a function of the difference between their mean distance moduli and the ones listed in Table 1. The results from fits to $B V R I$ data are plotted with open symbols, while those from griz data are shown by the filled symbols. The color and the shape of the symbols encode the fitting method as indicated by the figure legend.

From this plot it is seen that a separation of $\Delta \mu_{0} \sim 0.2$ mag can still be present between the distance moduli given by different LC-fitting codes, similar to the case of SN 2011fe, although the difference varies from SN to SN and it is less than $\sim 0.2$ mag for the majority of the cases considered in the present paper.

For the three moderately reddened SNe (2012cg, 2012ht and 2013dy) the distances given by MLCS2k2 and SALT2.4 are in remarkable agreement (their difference is $0.02,0.04$ and $-0.01 \mathrm{mag}$, respectively) for the $B V R I$ data. The differences between the MLCS2k2 and SNooPy2 distances in $B V R I$ are also very good; they do not exceed $0.1 \mathrm{mag}$. This is not true in the case of SN 2014J, as expected, because SALT2.4 could not provide reliable distances for such an extremely reddened object, thus, they are not plotted in Fig. 6. Note that this does not mean that SALT2.4 is a less reliable 

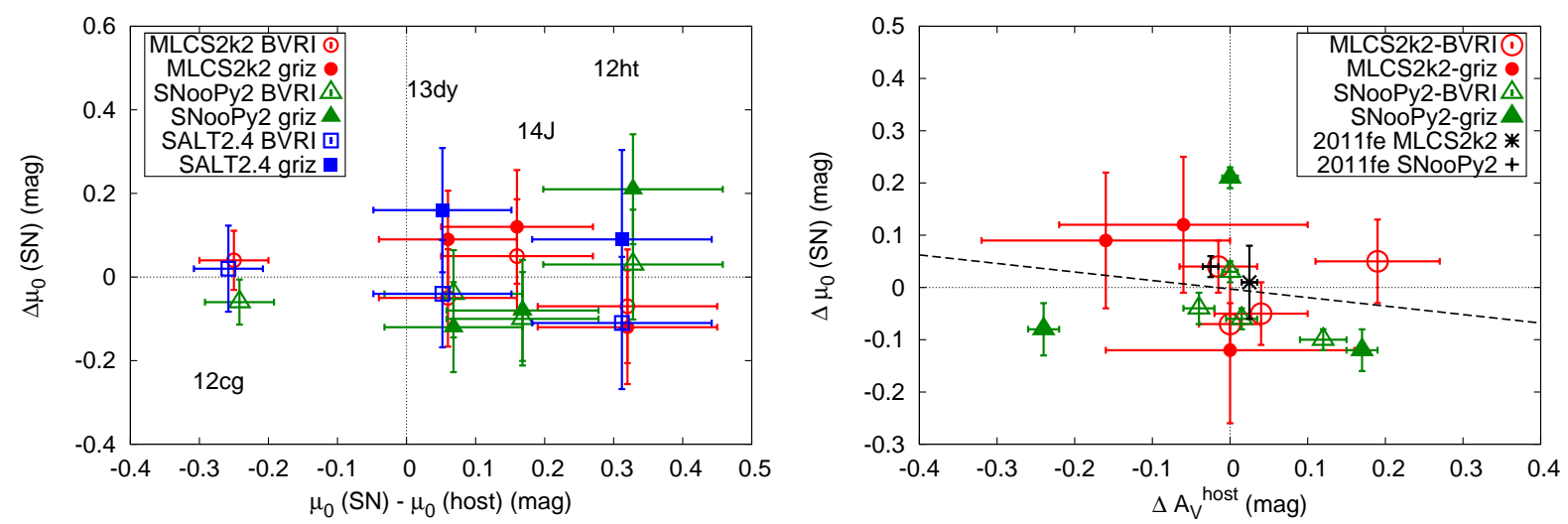

Figure 6. Left panel: Residual distance moduli from their mean value for each SNe. Different symbols denote the different LC-fitting codes, as explained in the legend. Right panel: the distance modulus residuals as a function of the dust extinction residuals. The dashed line indicates a simple linear fit to these data. See text for explanation.

Table 9. Final absolute distances to SNe Ia

\begin{tabular}{lccccc}
\hline \hline SN & $\begin{array}{c}\mu_{0}(\mathrm{SN}) \\
(\mathrm{mag})\end{array}$ & $\begin{array}{c}\mu_{0} \text { (host) } \\
(\mathrm{mag})\end{array}$ & $\begin{array}{c}D_{S N} \\
(\mathrm{Mpc})\end{array}$ & $\begin{array}{c}D_{\text {host }} \\
(\mathrm{Mpc})\end{array}$ & $\begin{array}{c}\text { Difference } \\
(\mathrm{mag})\end{array}$ \\
\hline $2011 \mathrm{fe}$ & $29.13 \pm 0.08$ & $29.13 \pm 0.04$ & $6.7_{-0.2}^{+0.3}$ & $6.7_{0.1}^{0.1}$ & $0.00(<1 \sigma)$ \\
$2012 \mathrm{cg}$ & $30.83 \pm 0.05$ & $31.08 \pm 0.29$ & $14.6_{-0.3}^{+0.3}$ & $16.44_{-2.1}^{+2.4}$ & $-0.25(\lesssim 1 \sigma)$ \\
$2012 \mathrm{ht}$ & $32.23 \pm 0.13$ & $31.91 \pm 0.04$ & $27.9_{-1.6}^{+1.7}$ & $24.1_{-0.5}^{+0.5}$ & $0.32(\sim 2.3 \sigma)$ \\
$2013 \mathrm{dy}$ & $31.54 \pm 0.08$ & $31.50 \pm 0.08$ & $20.3_{-0.7}^{+0.8}$ & $20.0_{-0.7}^{+0.8}$ & $0.04(\lesssim 1 \sigma)$ \\
$2014 \mathrm{~J}$ & $28.09 \pm 0.11$ & $27.93 \pm 0.35$ & $4.1_{-0.2}^{+0.2}$ & $3.9_{-0.6}^{+0.7}$ & $0.16(\lesssim 1 \sigma)$ \\
\hline
\end{tabular}

code. It is just the consequence of the underlying model, and the code works fine for moderately reddened SNe Ia.

The agreement is slightly worse for our $g^{\prime} r^{\prime} i^{\prime} z^{\prime}$ photometry, partly because those data have smaller signal-to-noise ratio than our $B V R I$ light curves. From these data it is found that the differences between the distance moduli provided by the three codes may differ by $\sim 0.2 \mathrm{mag}$. A similar $\lesssim 0.2$ mag difference can be seen when comparing the distances of the same SN taken from $B V R I$ and $g^{\prime} r^{\prime} i^{\prime} z^{\prime} \mathrm{LCs}$ : for SN 2012ht $\mu_{0}(B V R I)-\mu_{0}\left(g^{\prime} r^{\prime} i^{\prime} z^{\prime}\right)$ is $0.05,-0.18$ and -0.30 mag from MLCS2k2, SNooPy2 and SALT2.4, respectively; for SN 2013dy these are $-0.14,0.08$ and -0.20 ; for SN 2014J $-0.07,-0.02$ and -1.06 mag. It is seen that there is no systematic trend in these numbers, which suggests that these differences are probably not due to systematic errors in the photometric calibration; more probably they represent the internal uncertainties of the template LC vectors for the different bands. Given that our data were obtained by two telescopes from two different sites (one for $B V R I$ and another one for $g^{\prime} r^{\prime} i^{\prime} z^{\prime}$ ), this result may also give a hint on the possible amount of systematic errors in the distance moduli when fitting inhomogeneous LCs taken by more than one telescope.
It is concluded that applying these three popular LC-fitting codes as distance calculators on homogeneous $B V R I$ photometry on nearby, moderately reddened SNe Ia one can derive consistent distances that agree with each other within $\lesssim 0.1 \mathrm{mag}$ or better. Even for strongly reddened SNe, like SN 2014J, MLCS2k2 and SNooPy2 work quite well; their distances differ by only $\sim 0.15 \mathrm{mag}$. We found slightly larger ( $\sim 0.2 \mathrm{mag}$ ) differences in the distances from our $g^{\prime} r^{\prime} i^{\prime} z^{\prime}$ photometry.

In the right panel of Fig. 6 we investigate whether the differences between the distance moduli were due to systematic under- or overestimates of the extinction parameter $A_{V}^{\text {host }}$. In this panel the same $\Delta \mu_{0}$ residuals are plotted against the residual of the host extinction parameter $\Delta A_{V}^{\text {host }}$ (we consider only the extinction within the host here, since the Milky Way extinction was kept fixed at the values provided by the Milky Way dust maps). SN $2011 \mathrm{fe}$ is also plotted in this diagram (with black symbols) taking the data from Vinkó et al. (2012). Again, only the MLCS2k2 and SNooPy2 results are used.

If the expected correlation between the distances and extinction (higher extinction estimates imply shorter distances) exists, then one should see positive $\Delta \mu_{0}$ values for negative $\Delta A_{v}^{\text {host }}$ and vice versa. It is not clearly visible in Fig. 6, as 
most of the data scatter around 0 within $\sim 0.2$ mag in all directions. The dashed line is a simple linear fit to the data. Its slope, $-0.16 \pm 0.23$, is in the expected direction of a correlation between the extinction and distance, though it is not significant. Although it is probable that our sample is too low to detect this, the lack of significant correlation suggests that the distances given by either MLCS2k2 or SNooPy 2 are less affected by systematic under- or overestimates of the extinction parameter.

In Table 9 we summarize the final absolute distances for each SN studied, supplemented by the data for SN $2011 \mathrm{fe}$ from Vinkó et al. (2012). These are defined as the simple, unweighted mean of all distance moduli from our Konkoly + Baja data except for SN 2014J where the SALT2.4 distances were omitted due to the reason mentioned above. The Cepheid-based distances to the host galaxies (Riess et al. 2016) from Table 1 are also shown for easy comparison. In the final column the difference between the SN and host distance moduli is given with respect to their combined uncertainties $\sigma\left(\mu_{0}\right)=\sqrt{\sigma_{S N}^{2}+\sigma_{\text {host }}^{2}}$. It is apparent that even though the individual SN-based mean distances are uncertain at the $\sim 0.10$ mag level, their deviations from their host galaxy distances obtained independently are less than $1 \sigma$ in 4 out of 5 cases, which is encouraging. Higher deviation $(2 \sigma)$ is seen only in the case of SN 2012ht.

Recently Riess et al. (2016) showed that by properly combining Cepheid- and SN Ia-based distance scales, anchored to local galaxies having independent geometric distances, one can reduce the uncertainty of the local value of the Hubbleconstant $\left(H_{0}\right)$ to $\sim 2.4$ percent. Such an accuracy on the individual distances to local galaxies would need $\mathrm{a} \sim 0.05 \mathrm{mag}$ dispersion in the distance moduli. Our results above show that this is still not the case for every $\mathrm{SN}$ at present, although the agreement between the distance moduli are close to the $\lesssim 0.05$ mag level for the best-observed SNe in our sample. The $\sim 0.1-0.2$ mag dispersion between the distance moduli calculated by different LC fitters is close to the $\sim 0.15 \mathrm{mag}$ dispersion found by Riess et al. (2016) when comparing their Cepheid- and SN Ia-based distances to the same galaxies.

As the sample of the SNe Ia having accurately calibrated photometry (Scolnic et al. 2015) is growing rapidly, we can expect significant improvement in the accuracy of the individual distance estimates to local galaxies in the near future. This would be an important step toward better understanding the physics of SN Ia explosions.

\section{SUMMARY}

We have studied 3 public light curve fitting codes for SNe Ia by cross-comparing the time of maximum, extinction and distance parameters inferred from the light curves of 4 nearby, bright, well-observed SNe Ia (2012cg, 2012ht, $2013 \mathrm{dy}, 2014 \mathrm{~J})$. Our results are summarized as follows.
- The moment of $B$-band maximum can be estimated within \pm 0.7 day, even if there are no data observed in the $B$-band. Note that SALT2.4 tends to give systematically later maximum times by $\Delta t_{\max } \sim 0.5$ day than MLCS2k2 (Vinkó et al. 2012; Pereira et al. 2013).

- For moderately reddened $\left(A_{V}^{\text {host }}<0.5 \mathrm{mag}\right) \mathrm{SNe}$, MLCS2k2 and SNooPy2 did quite a good job in estimating the relative amount of interstellar extinction within the host galaxy ( $A_{V}^{\text {host }}$ ) compared to the extinction within the Milky Way $\left(A_{V}^{M W}\right)$. The inferred $A_{V}^{\text {host }} / A_{V}^{M W}$ extinction ratios for SNe 2012ht and 2013 dy are consistent with the relative strengths of the interstellar $\mathrm{NaD}$ lines from high-resolution spectroscopy. This is not the case for the heavily-reddened SN 2014J, where the light curve fitting resulted in reddening parameters having large scatter and being different from the results of spectroscopic analyses.

- Regarding the distance modulus, it is found that for the moderately reddened SNe 2012cg, 2012ht and 2013dy the consistency between the results from the three LC-fitting codes is $\lesssim 0.1 \mathrm{mag}$ for our highest quality $B V R I$ data, even without taking into account the dependence of the peak brightnesses on the host galaxy masses. This is significantly better than the $\sim 0.16$ mag difference found by Vinkó et al. (2012) for SN 2011fe. The dispersion is somewhat higher, $\sim 0.2 \mathrm{mag}$, for our $g^{\prime} r^{\prime} i^{\prime} z^{\prime} \mathrm{LCs}$ that have lower S/N ratio, and the same is true for the strongly reddened SN 2014J. We found a negative, though nonsignificant, distance-extinction correlation in our sample, suggesting that the distances provided by both MLCS2k2 and SNooPy 2 are not strongly affected by systematic overor underestimates of the extinction. The final distances to our sample $\mathrm{SNe}$ are in very good agreement with the Cepheid-based distances to their host galaxies (see Table 9). From these results it seems important to utilize only low-extinction $\left(A_{V}<0.5 \mathrm{mag}\right) \mathrm{SNe}$ Ia to measure $H_{0}$ in order to reduce the potential systematic errors on the derived distances due to dust extinction (Riess et al. 2016).

We are indebted to Adam Riess for his valuable comments and suggestions on the previous version of this paper. We also thank the thorough work of an anonymous referee for his/her report that helped significantly to improve the paper. This work is part of the project "Transient Astrophysical Objects" GINOP 2.3.2-15-2016-00033 of the National Research, Development and Innovation Office (NKFIH), Hungary, funded by the European Union. Some co-authors of this publication have also received funds from the follow- 
ing grants: Hungarian NKFIH/OTKA PD-116175 (LM), PD112325 (TS), K-109276 (KV), K-113117 (KV) and NN107637 (JV); MTA-Lendulet LP2012-31 (AP); NSF Fellowship AST-1302771 (JMS); NSF Grant AST-1109881 (JCW).
JCW is supported by the Samuel T. and Fern Yanagisawa Regents Professorship. LM and KV are supported by the Bolyai Janos Research Scholarship of the Hungarian Academy of Sciences.

\section{REFERENCES}

Amanullah, R., Goobar, A., Johansson, J., et al. 2014, ApJL, 788, L21

Amanullah, R., Johansson, J., Goobar, A., et al. 2015, MNRAS, 453, 3300

Astier, P., Guy, J., Regnault, N., et al. 2006, A\&A, 447, 31

Benitez-Herrera, S., Ishida, E. E. O., Maturi, M., et al. 2013, MNRAS, 436, 854

Betoule, M., Kessler, R., Guy, J., et al. 2014, A\&A, 568, A22

Brown, P. J., Smitka, M. T., Wang, L., et al. 2015, ApJ, 805, 74

Burns, C. R., Stritzinger, M., Phillips, M. M., et al. 2011, AJ, 141, 19

Burns, C. R., Stritzinger, M., Phillips, M. M., et al. 2014, ApJ, 789, 32

Cardelli, J. A., Clayton, G. C., \& Mathis, J. S. 1989, ApJ, 345, 245

Conley, A., Sullivan, M., Hsiao, E. Y., et al. 2008, ApJ, 681, 482-498

Conley, A., Guy, J., Sullivan, M., et al. 2011, ApJS, 192, 1

Cortés, J. R., Kenney, J. D. P., \& Hardy, E. 2006, AJ, 131, 747

Dale, D. A., Gil de Paz, A., Gordon, K. D., et al. 2007, ApJ, 655, 863

Dhawan, S., Jha, S. W., \& Leibundgut, B. 2017, arXiv:1707.00715

Draine, B. T. 2003, ARA\&A, 41, 241

Folatelli, G., Phillips, M. M., Burns, C. R., et al. 2010, AJ, 139, 120

Foley, R. J., Fox, O. D., McCully, C., et al. 2014, MNRAS, 443, 2887

Fossey, S. J., Cooke, B., Pollack, G., Wilde, M., \& Wright, T. 2014, Central Bureau Electronic Telegrams, 3792, 1

Friedman, A. S., Wood-Vasey, W. M., Marion, G. H., et al. 2015, ApJS, 220, 9

Gao, J., Jiang, B. W., Li, A., Li, J., \& Wang, X. 2015, ApJL, 807, L26

Goobar, A., Johansson, J., Amanullah, R., et al. 2014, ApJL, 784, L12

Guy, J., Astier, P., Baumont, S., et al. 2007, A\&A, 466, 11

Guy, J., Sullivan, M., Conley, A., et al. 2010, A\&A, 523, A7

Hicken, M., Wood-Vasey, W. M., Blondin, S., et al. 2009, ApJ, 700, 1097

Hsiao, E. Y., Conley, A., Howell, D. A., et al. 2007, ApJ, 663, 1187 Jha, S., Garnavich, P. M., Kirshner, R. P., et al. 1999, ApJS, 125, 73 Jha, S., Riess, A. G., \& Kirshner, R. P. 2007, ApJ, 659, 122 Jordi, K., Grebel, E. K., \& Ammon, K. 2006, A\&A, 460, 339

Kelly, P. L., Hicken, M., Burke, D. L., Mandel, K. S., \& Kirshner, R. P. 2010, ApJ, 715, 743
Kessler, R., Becker, A. C., Cinabro, D., et al. 2009, ApJS, 185, 32 Lampeitl, H., Smith, M., Nichol, R. C., et al. 2010, ApJ, 722, 566

Le Borgne, D., \& Rocca-Volmerange, B. 2002, A\&A, 386, 446

Mandel, K. S., Narayan, G., \& Kirshner, R. P. 2011, ApJ, 731, 120

Marion, G. H., Sand, D. J., Hsiao, E. Y., et al. 2015, ApJ, 798, 39

Marion, G. H., Brown, P. J., Vinkó, J., et al. 2015, arXiv:1507.07261

Mazzei, P., Marino, A., Rampazzo, R., et al. 2017, arXiv: 1710.07474

Mosher, J., Guy, J., Kessler, R., et al. 2014, ApJ, 793, 16

Munari, U., Henden, A., Belligoli, R., et al. 2013, NewA, 20, 30

Pan, Y.-C., Sullivan, M., Maguire, K., et al. 2014, MNRAS, 438, 1391

Pan, Y.-C., Foley, R. J., Kromer, M., et al. 2015, MNRAS, 452, 4307

Pereira, R., Thomas, R. C., Aldering, G., et al. 2013, A\&A, 554, A27

Perlmutter, S., Aldering, G., Goldhaber, G., et al. 1999, ApJ, 517, 565

Phillips, M. M. 1993, ApJL, 413, L105

Planck Collaboration, Ade, P. A. R., Aghanim, N., et al. 2014, A\&A, 571, A16

Planck Collaboration, Ade, P. A. R., Aghanim, N., et al. 2015, arXiv:1502.01589

Poznanski, D., Prochaska, J. X., \& Bloom, J. S. 2012, MNRAS, 426, 1465

Prieto, J. L., Rest, A., \& Suntzeff, N. B. 2006, ApJ, 647, 501

Pskovskii, I. P. 1977, AZh, 54, 1188

Rest, A., Scolnic, D., Foley, R. J., et al. 2014, ApJ, 795, 44

Richmond, M. W., Treffers, R. R., Filippenko, A. V., et al. 1994, AJ, 107, 1022

Riess, A. G., Filippenko, A. V., Challis, P., et al. 1998, AJ, 116, 1009

Riess, A. G., Li, W., Stetson, P. B., et al. 2005, ApJ, 627, 579

Riess, A. G., Strolger, L.-G., Casertano, S., et al. 2007, ApJ, 659, 98

Riess, A. G., Macri, L., Casertano, S., et al. 2011, ApJ, 730, 119

Riess, A. G., Macri, L. M., Hoffmann, S. L., et al. 2016, ApJ, 826, 56

Saunders, C., Aldering, G., Antilogus, P., et al. 2015, ApJ, 800, 57

Schlafly, E. F., \& Finkbeiner, D. P. 2011, ApJ, 737, 103

Scolnic, D., Rest, A., Riess, A., et al. 2014, ApJ, 795, 45

Scolnic, D., Casertano, S., Riess, A., et al. 2015, ApJ, 815, 117 
Scolnic, D., \& Kessler, R. 2016, ApJL, 822, L35

Shariff, H., Dhawan, S., Jiao, X., et al. 2016, MNRAS, 463, 4311

Silverman, J. M., Ganeshalingam, M., Cenko, S. B., et al. 2012, ApJL, 756, L7

Sullivan, M., Conley, A., Howell, D. A., et al. 2010, MNRAS, 406, 782

Vinkó, J. et al. 2012, A\&A546, 12

Walker, E. S., Baltay, C., Campillay, A., et al. 2015, ApJS, 219, 13

Weyant, A., Wood-Vasey, W. M., Joyce, R., et al. 2017, arXiv:1703.02402
Wood-Vasey, W. M., Miknaitis, G., Stubbs, C. W., et al. 2007, ApJ, 666, 694

Yamanaka, M., Maeda, K., Kawabata, M., et al. 2014, ApJL, 782, L35

Yusa, T., Itagaki, K., Nakano, S., et al. 2012, Central Bureau Electronic Telegrams, 3349, 1

Zhai, Q., Zhang, J.-J., Wang, X.-F., et al. 2016, AJ, 151, 125

Zhang, B. R., Childress, M. J., Davis, T. M., et al. 2017, MNRAS, 471, 2254

Zheng, W., Silverman, J. M., Filippenko, A. V., et al. 2013, ApJL, 778, L15

Zheng, W., Shivvers, I., Filippenko, A. V., et al. 2014, ApJL, 783, L24 

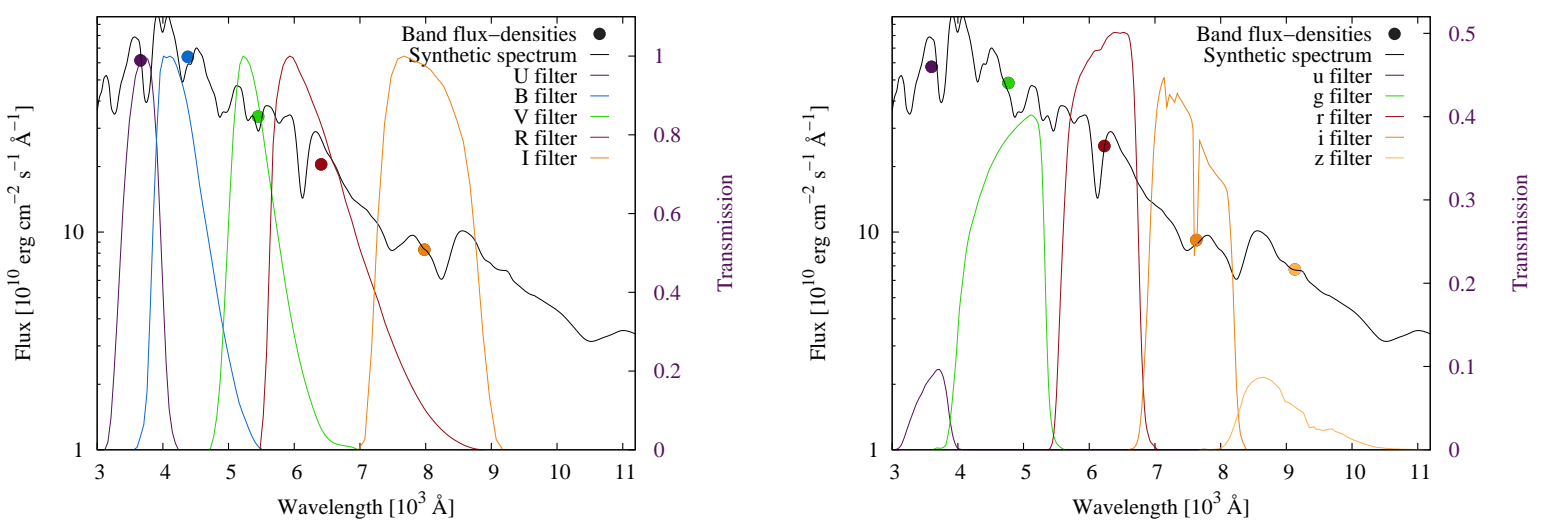

Figure 7. Left panel: The Hsiao template spectrum at maximum light and the transmission curves of the Johnson-Cousins $U B V R I$ system. Filled symbols indicate the synthetic flux densities. Right panel: the same but for the Sloan $u^{\prime} g^{\prime} r^{\prime} i^{\prime} z^{\prime}$ system.

\section{APPENDIX}

\section{A. THE CONSTRUCTION OF $G^{\prime} R^{\prime} I^{\prime} Z^{\prime}$ VECTORS FOR MLCS2K2}

Since MLCS2k2 is originally designed for only the $U B V R I$ system (Jha, Riess \& Kirshner 2007), one needs to transform its $M_{X}, P_{X}$ and $Q_{X}$ vectors $(X=\{U, B, V, R, I\})$ to other filters if data from other photometric system are to be fit. We have computed the transformation to the Sloan $u^{\prime} g^{\prime} r^{\prime} i^{\prime} z^{\prime}$ system via the Hsiao template spectra (Hsiao et al. 2007) in the following way.

First, synthetic fluxes for both $U B V R I$ and $u^{\prime} g^{\prime} r^{\prime} i^{\prime} z^{\prime}$ filters were computed from the Hsiao template spectra by convolving the templates with the corresponding filter functions (Fig.7). This was done for all templates between -10 day and +90 day. Next, the following flux ratios were defined as the basis of the $U B V R I \rightarrow u^{\prime} g^{\prime} r^{\prime} i^{\prime} z^{\prime}$ transformation: $f\left(u^{\prime}\right) / f(U), f\left(g^{\prime}\right) / f(B)$, $f\left(r^{\prime}\right) / f(R), f\left(i^{\prime}\right) / f(I)$ and $f\left(z^{\prime}\right) / f(I)$. Among many other combinations, these flux ratios were found to exhibit the least amount of variation in the $[-10 \mathrm{~d},+90 \mathrm{~d}]$ phase interval. Third, using these flux ratios the MLCS2k $U B V R I$ magnitudes were transformed to fluxes, multiplied by the corresponding flux ratios and converted back to Sloan $u^{\prime} g^{\prime} r^{\prime} i^{\prime} z^{\prime}$ magnitudes. Note that this conversion is based on the implicit assumption that the above flux ratios remain the same for SNe having different MLCS2k2 $\Delta$ parameter. This certainly breaks down for SNe having large $(>0.5) \Delta$; however, since our SNe have only $\Delta$ within \pm 0.3 , this assumption should be approximately valid. The feasibility of the whole transformation can also be judged by the consistency between the MLCS2k2 fitting parameters computed from the quasi-simultaneous $U B V R I$ and $u^{\prime} g^{\prime} r^{\prime} i^{\prime} z^{\prime}$ data.

MLCS2k2 also contains a prescription for the time-dependence of the extinction in the $U B V R I$ bands, parametrized as $\zeta_{X}(t)=A_{X}(t) / A_{X}\left(t_{B \max }\right)$, where $A_{X}$ is the extinction in the $X$-band. We transformed the $\zeta_{X}$ vectors of Jha, Riess \& Kirshner (2007) to the $u^{\prime} g^{\prime} r^{\prime} i^{\prime} z^{\prime}$ system using a similar approach as above. First, following Jha, Riess \& Kirshner (2007), the Hsiao templates were reddened with a chosen $A_{V}$ and using the Cardelli-law (Cardelli et al. 1989):

$$
\frac{A(x)}{A_{V}}=\left(\alpha(x)+\frac{\beta(x)}{R_{V}}\right) .
$$

The standard Milky Way reddening slope, $R_{V}=3.1$, was assumed at first, but this condition was relaxed later (see below).

Next, both the reddened and the reddening-free $U B V R I$ magnitudes were transformed to $u^{\prime} g^{\prime} r^{\prime} i^{\prime} z^{\prime}$ magnitudes as above, and their time-dependent differences were used to construct the $\zeta$ vectors for the Sloan system: $\zeta_{X}=\left(M_{X}^{\prime}(t)-\right.$ $\left.M_{X}(t)\right) /\left(M_{X}^{\prime}\left(t_{B \max }\right)-M_{X}\left(t_{B \max }\right)\right)$, where $M_{X}^{\prime}$ denotes the reddened MLCS2k2 magnitude in the $X$-band.

To be able to handle non-standard (i.e. $R_{V} \neq 3.1$ ) reddening slopes, the procedure described above has been repeated for $1<R_{V}<6$. It was found that the resulting $\zeta_{X}\left(R_{V}\right)$ values as a function of $1-\zeta_{X}(3.1)$ can be approximated by a parabola of the following form:

$$
1-\zeta_{X}\left(R_{V}\right)=a_{X} \cdot\left(1-\zeta_{X}(3.1)\right)^{2}+b_{X} \cdot\left(1-\zeta_{X}(3.1)\right)+c_{X}
$$

Since the $a_{X}, b_{X}$ and $c_{X}$ coefficients are different for every $R_{V}$, their dependency on $R_{V}$ were determined by fitting a cubic polynomial to these data as a function of $R_{V}^{-1}$ :

$$
a_{X}=d_{1,1} \cdot\left(R_{V}^{-1}\right)^{3}+d_{1,2} \cdot\left(R_{V}^{-1}\right)^{2}+d_{1,3} \cdot\left(R_{V}^{-1}\right)+d_{1,4}
$$


Table 10. The constant parameters from the third-order polynomial fit to $a_{X}, b_{X}$ and $c_{X}$ as functions of $R_{V}^{-1}$ (see Eq.A3).

\begin{tabular}{lcccccccccc}
\hline \hline & $U$ & $B$ & $V$ & $R$ & $I$ & $u^{\prime}$ & $g^{\prime}$ & $r^{\prime}$ & $i^{\prime}$ & $z^{\prime}$ \\
\hline$d_{1,1}$ & -29.637 & 1.711 & 0.355 & -1.157 & -11.212 & -41.028 & 4.925 & -0.974 & -8.647 & -193.074 \\
$d_{2,1}$ & 2.790 & 0.845 & 0.023 & 0.235 & 5.962 & 2.828 & 0.424 & 0.120 & -1.468 & 13.272 \\
$d_{3,1} \cdot 10^{-3}$ & 0.149 & -0.222 & 0.053 & 0.157 & -6.828 & -0.126 & 1.938 & 0.028 & -0.718 & 44.466 \\
$d_{1,2}$ & 81.694 & -5.761 & -0.618 & -1.431 & 9.372 & 108.431 & -9.990 & -0.590 & 0.074 & 218.568 \\
$d_{2,2}$ & -7.388 & -2.551 & -0.236 & 0.339 & -4.992 & -5.476 & -1.879 & 0.268 & 6.286 & -15.035 \\
$d_{3,2} \cdot 10^{-3}$ & -0.443 & 0.777 & -0.676 & 0.215 & 5.714 & -1.256 & -3.057 & 0.051 & -0.621 & -50.453 \\
$d_{1,3}$ & -77.845 & -0.101 & -2.496 & -3.137 & -6.392 & -79.613 & 5.363 & 6.273 & 1.242 & -91.518 \\
$d_{2,3}$ & 7.736 & 4.200 & 2.503 & 1.409 & 3.171 & 6.257 & 3.709 & 1.259 & -2.521 & 6.292 \\
$d_{3,3} \cdot 10^{-3}$ & 0.401 & 0.409 & 1.466 & 1.314 & -3.743 & 0.560 & 0.973 & 0.785 & 0.378 & 21.295 \\
$d_{1,4}$ & 17.612 & 0.573 & 0.855 & 1.199 & 1.443 & 15.798 & -0.897 & -1.937 & -0.173 & 12.752 \\
$d_{2,4}$ & -0.822 & -0.118 & 0.216 & 0.502 & 0.306 & -0.545 & -0.013 & 0.562 & 1.256 & 0.124 \\
$d_{3,4} \cdot 10^{-3}$ & -0.088 & -0.205 & -0.404 & -0.452 & 0.831 & -0.046 & -0.084 & -0.259 & -0.043 & -2.997 \\
\hline \hline
\end{tabular}

$$
\begin{aligned}
& b_{X}=d_{2,1} \cdot\left(R_{V}^{-1}\right)^{3}+d_{2,2} \cdot\left(R_{V}^{-1}\right)^{2}+d_{2,3} \cdot\left(R_{V}^{-1}\right)+d_{2,4} \\
& c_{X}=d_{3,1} \cdot\left(R_{V}^{-1}\right)^{3}+d_{3,2} \cdot\left(R_{V}^{-1}\right)^{2}+d_{3,3} \cdot\left(R_{V}^{-1}\right)+d_{3,4}
\end{aligned}
$$

The fit parameters for both photometric systems are collected in Table 10. 
Table 11. Local $B V R I$ standard stars in the vicinity of SN $2012 \mathrm{cg}$

\begin{tabular}{cccccccccc}
\hline \hline R.A. & Dec. & $\mathrm{B}$ & $\sigma_{B}$ & $\mathrm{~V}$ & $\sigma_{V}$ & $\mathrm{R}$ & $\sigma_{R}$ & $\mathrm{I}$ & $\sigma_{I}$ \\
\hline 12:27:28.9 & $9: 29: 33.6$ & 16.241 & 0.011 & 15.648 & 0.013 & 15.345 & 0.011 & 14.932 & 0.017 \\
$12: 26: 48.0$ & $9: 28: 50.5$ & 15.408 & 0.011 & 14.644 & 0.013 & 14.245 & 0.011 & 13.771 & 0.017 \\
$12: 26: 48.3$ & $9: 29: 57.3$ & 15.805 & 0.011 & 14.998 & 0.013 & 14.570 & 0.011 & 14.050 & 0.017 \\
$12: 27: 15.9$ & $9: 27: 27.0$ & 15.046 & 0.011 & 14.506 & 0.013 & 14.232 & 0.011 & 13.841 & 0.017 \\
$12: 27: 29.8$ & $9: 23: 53.2$ & 15.919 & 0.011 & 15.078 & 0.013 & 14.633 & 0.011 & 14.106 & 0.017 \\
\hline \hline
\end{tabular}

Table 12. Photometry of SN 2012cg. The $B V R I$ data are given in Vega-magnitudes. Errors are given in parentheses.

\begin{tabular}{ccccc}
\hline \hline MJD & $\mathrm{B}$ & $\mathrm{V}$ & $\mathrm{R}$ & $\mathrm{I}$ \\
\hline 56066.8 & $14.995(0.031)$ & $14.679(0.025)$ & $14.593(0.023)$ & $14.420(0.048)$ \\
56067.9 & $14.493(0.014)$ & $14.221(0.010)$ & $14.133(0.011)$ & $13.974(0.018)$ \\
56069.8 & $13.629(0.014)$ & $13.490(0.010)$ & $13.375(0.009)$ & $13.229(0.018)$ \\
56072.9 & $12.765(0.008)$ & $12.674(0.010)$ & $12.575(0.008)$ & $12.462(0.017)$ \\
56073.8 & $12.646(0.015)$ & $12.475(0.016)$ & $12.481(0.012)$ & $12.360(0.019)$ \\
56075.8 & $12.452(0.012)$ & $12.175(0.012)$ & $12.233(0.007)$ & $12.179(0.017)$ \\
56076.8 & $12.141(0.017)$ & $12.172(0.011)$ & $12.108(0.011)$ & $12.110(0.022)$ \\
56077.8 & $12.205(0.011)$ & $12.150(0.009)$ & $12.105(0.009)$ & $12.061(0.017)$ \\
56079.9 & $12.132(0.008)$ & $12.018(0.010)$ & $11.994(0.008)$ & $12.032(0.015)$ \\
56080.8 & $12.150(0.013)$ & $11.995(0.012)$ & $11.954(0.009)$ & $12.058(0.018)$ \\
56081.8 & $12.097(0.017)$ & $11.951(0.015)$ & $11.950(0.012)$ & $12.055(0.022)$ \\
56084.9 & $12.139(0.010)$ & $11.904(0.013)$ & $11.888(0.012)$ & $12.097(0.023)$ \\
56089.9 & $12.439(0.016)$ & $12.055(0.023)$ & $12.042(0.024)$ & $12.352(0.030)$ \\
56092.9 & $12.617(0.018)$ & $12.258(0.010)$ & $12.296(0.008)$ & $12.540(0.019)$ \\
56093.9 & $12.726(0.018)$ & $12.325(0.021)$ & $12.354(0.012)$ & $12.606(0.032)$ \\
56094.9 & $12.799(0.025)$ & $12.355(0.020)$ & $12.441(0.013)$ & $12.635(0.034)$ \\
56095.9 & $12.918(0.033)$ & $12.462(0.021)$ & $12.502(0.014)$ & $12.659(0.031)$ \\
56097.9 & $13.143(0.032)$ & $12.576(0.022)$ & $12.550(0.018)$ & $12.659(0.032)$ \\
56098.9 & $13.251(0.027)$ & $12.629(0.022)$ & $12.611(0.010)$ & $12.647(0.028)$ \\
56099.9 & $13.389(0.025)$ & $12.677(0.021)$ & $12.644(0.017)$ & $12.630(0.031)$ \\
56101.9 & $13.565(0.025)$ & $12.834(0.020)$ & $12.666(0.010)$ & $12.607(0.027)$ \\
56102.9 & $13.756(0.029)$ & $12.884(0.023)$ & $12.640(0.018)$ & $12.571(0.031)$ \\
56104.9 & $13.992(0.032)$ & $12.951(0.026)$ & $12.728(0.013)$ & $12.568(0.032)$ \\
\hline \hline & & & &
\end{tabular}

Table 13. Local $B V R I$ standard stars in the vicinity of SN 2012ht

\begin{tabular}{cccccccccc}
\hline \hline R.A. & Dec. & B & $\sigma_{B}$ & $\mathrm{~V}$ & $\sigma_{V}$ & $\mathrm{R}$ & $\sigma_{R}$ & $\mathrm{I}$ & $\sigma_{I}$ \\
\hline 10:53:01.648 & $+16: 56: 03.06$ & 14.886 & 0.009 & 14.225 & 0.011 & 13.787 & 0.014 & 13.405 & 0.007 \\
10:54:06.561 & $+16: 45: 53.13$ & 15.589 & 0.005 & 14.944 & 0.006 & 14.697 & 0.005 & 14.505 & 0.009 \\
10:53:39.512 & $+16: 49: 17.04$ & 15.914 & 0.005 & 15.300 & 0.006 & 14.991 & 0.005 & 14.575 & 0.006 \\
10:54:01.309 & $+16: 50: 07.90$ & 15.759 & 0.005 & 15.149 & 0.006 & 14.841 & 0.005 & 14.426 & 0.006 \\
10:52:54.642 & $+16: 59: 01.96$ & 15.253 & 0.010 & 14.524 & 0.013 & 13.995 & 0.016 & 13.555 & 0.008 \\
10:53:05.252 & $+16: 50: 56.91$ & 15.838 & 0.005 & 15.149 & 0.006 & 14.799 & 0.005 & 14.354 & 0.006 \\
10:54:01.439 & $+16: 51: 57.31$ & 16.054 & 0.005 & 15.160 & 0.006 & 14.686 & 0.005 & 14.107 & 0.005 \\
10:52:57.121 & $+16: 54: 56.27$ & 15.751 & 0.006 & 14.975 & 0.007 & 14.497 & 0.007 & 13.958 & 0.007 \\
\hline \hline
\end{tabular}

\section{B. PHOTOMETRIC DATA}


Table 14. Photometry of SN 2012ht. The $B V R I$ data are given in Vega-magnitudes, while the $g^{\prime} r^{\prime} i^{\prime} z^{\prime}$ data are in AB-magnitudes.

\begin{tabular}{|c|c|c|c|c|c|c|c|c|}
\hline MJD & $\mathrm{B}$ & $\sigma_{B}$ & $\mathrm{~V}$ & $\sigma_{V}$ & $\mathrm{R}$ & $\sigma_{R}$ & I & $\sigma_{I}$ \\
\hline 56066.8 & 14.995 & 0.031 & 14.679 & 0.025 & 14.593 & 0.023 & 14.420 & 0.048 \\
\hline 56067.9 & 14.493 & 0.014 & 14.221 & 0.010 & 14.133 & 0.011 & 13.974 & 0.018 \\
\hline 56069.8 & 13.629 & 0.014 & 13.490 & 0.010 & 13.375 & 0.009 & 13.229 & 0.018 \\
\hline 56072.9 & 12.765 & 0.008 & 12.674 & 0.010 & 12.575 & 0.008 & 12.462 & 0.017 \\
\hline 56073.8 & 12.646 & 0.015 & 12.475 & 0.016 & 12.481 & 0.012 & 12.360 & 0.019 \\
\hline 56075.8 & 12.452 & 0.012 & 12.175 & 0.012 & 12.233 & 0.007 & 12.179 & 0.017 \\
\hline 56076.8 & 12.141 & 0.017 & 12.172 & 0.011 & 12.108 & 0.011 & 12.110 & 0.022 \\
\hline 56077.8 & 12.205 & 0.011 & 12.150 & 0.009 & 12.105 & 0.009 & 12.061 & 0.017 \\
\hline 56079.9 & 12.132 & 0.008 & 12.018 & 0.010 & 11.994 & 0.008 & 12.032 & 0.015 \\
\hline 56080.8 & 12.150 & 0.013 & 11.995 & 0.012 & 11.954 & 0.009 & 12.058 & 0.018 \\
\hline 56081.8 & 12.097 & 0.017 & 11.951 & 0.015 & 11.950 & 0.012 & 12.055 & 0.022 \\
\hline 56084.9 & 12.139 & 0.010 & 11.904 & 0.013 & 11.888 & 0.012 & 12.097 & 0.023 \\
\hline 56089.9 & 12.439 & 0.016 & 12.055 & 0.023 & 12.042 & 0.024 & 12.352 & 0.030 \\
\hline 56092.9 & 12.617 & 0.018 & 12.258 & 0.010 & 12.296 & 0.008 & 12.540 & 0.019 \\
\hline 56093.9 & 12.726 & 0.018 & 12.325 & 0.021 & 12.354 & 0.012 & 12.606 & 0.032 \\
\hline 56094.9 & 12.799 & 0.025 & 12.355 & 0.020 & 12.441 & 0.013 & 12.635 & 0.034 \\
\hline 56095.9 & 12.918 & 0.033 & 12.462 & 0.021 & 12.502 & 0.014 & 12.659 & 0.031 \\
\hline 56097.9 & 13.143 & 0.032 & 12.576 & 0.022 & 12.550 & 0.018 & 12.659 & 0.032 \\
\hline 56098.9 & 13.251 & 0.027 & 12.629 & 0.022 & 12.611 & 0.010 & 12.647 & 0.028 \\
\hline 56099.9 & 13.389 & 0.025 & 12.677 & 0.021 & 12.644 & 0.017 & 12.630 & 0.031 \\
\hline 56101.9 & 13.565 & 0.025 & 12.834 & 0.020 & 12.666 & 0.010 & 12.607 & 0.027 \\
\hline 56102.9 & 13.756 & 0.029 & 12.884 & 0.023 & 12.640 & 0.018 & 12.571 & 0.031 \\
\hline 56104.9 & 13.992 & 0.032 & 12.951 & 0.026 & 12.728 & 0.013 & 12.568 & 0.032 \\
\hline MJD & $\mathrm{g}$ & $\sigma_{g}$ & $\mathrm{r}$ & $\sigma_{r}$ & $\mathrm{i}$ & $\sigma_{i}$ & $\mathrm{z}$ & $\sigma_{z}$ \\
\hline 56285.94 & 14.182 & 0.227 & 14.074 & 0.094 & 14.277 & 0.087 & - & - \\
\hline 56290.01 & 13.508 & 0.107 & 13.444 & 0.039 & 13.657 & 0.062 & 13.867 & 0.075 \\
\hline 56291.14 & 13.347 & 0.151 & 13.375 & 0.094 & 13.673 & 0.073 & 13.730 & 0.107 \\
\hline 56292.11 & 13.383 & 0.147 & 13.250 & 0.115 & 13.513 & 0.119 & 13.883 & 0.126 \\
\hline 56303.97 & 13.402 & 0.140 & 13.518 & 0.119 & 14.306 & 0.167 & 14.335 & 0.173 \\
\hline 56315.94 & 14.700 & 0.270 & 14.037 & 0.109 & 14.457 & 0.215 & 14.213 & 0.410 \\
\hline 56322.94 & 15.277 & 0.288 & 14.537 & 0.098 & 14.658 & 0.188 & 14.368 & 0.189 \\
\hline 56326.86 & 15.458 & 0.202 & 14.757 & 0.064 & 14.873 & 0.189 & 14.551 & 0.178 \\
\hline 56330.97 & 15.601 & 0.129 & 14.964 & 0.078 & 15.180 & 0.110 & 14.953 & 0.211 \\
\hline 56331.91 & 15.529 & 0.047 & 15.003 & 0.113 & 15.195 & 0.121 & 14.988 & 0.170 \\
\hline 56340.92 & 15.975 & 0.153 & 15.477 & 0.100 & 15.616 & 0.138 & 15.482 & 0.157 \\
\hline 56351.86 & 16.162 & 0.226 & 15.896 & 0.143 & 15.649 & 0.224 & 15.316 & 0.293 \\
\hline 56353.86 & 16.122 & 0.122 & 15.815 & 0.135 & 16.081 & 0.188 & 16.085 & 0.156 \\
\hline 56355.96 & 16.199 & 0.199 & 15.931 & 0.146 & 16.201 & 0.203 & 16.393 & 0.254 \\
\hline 56356.80 & 16.392 & 0.181 & 15.958 & 0.181 & 16.205 & 0.191 & 16.624 & 0.289 \\
\hline 56362.91 & 16.372 & 0.208 & 16.206 & 0.149 & 16.424 & 0.224 & 16.468 & 0.236 \\
\hline 56388.80 & 16.875 & 0.175 & 17.059 & 0.151 & 17.456 & 0.232 & 17.738 & 0.394 \\
\hline 56395.91 & 17.092 & 0.166 & 17.087 & 0.045 & 17.444 & 0.143 & 18.357 & 0.488 \\
\hline 56396.80 & 16.949 & 0.146 & 17.281 & 0.152 & 17.330 & 0.241 & 18.708 & 1.002 \\
\hline 56397.80 & 16.947 & 0.133 & 17.180 & 0.060 & 17.666 & 0.157 & 18.496 & 0.410 \\
\hline 56398.86 & 16.930 & 0.093 & 17.348 & 0.100 & 17.499 & 0.104 & 17.457 & 0.191 \\
\hline
\end{tabular}

Table 15. Local $B V R I$ standard stars in the vicinity of SN 2013dy

\begin{tabular}{cccccccccc}
\hline \hline R.A. & Dec. & В & $\sigma_{B}$ & $\mathrm{~V}$ & $\sigma_{V}$ & $\mathrm{R}$ & $\sigma_{R}$ & $\mathrm{I}$ & $\sigma_{I}$ \\
\hline 22:18:22.19 & $+40: 34: 21.9$ & 16.233 & 0.032 & 15.606 & 0.014 & 15.243 & 0.015 & 14.844 & 0.016 \\
22:18:16.96 & $+40: 34: 55.9$ & 15.539 & 0.033 & 14.927 & 0.011 & 14.584 & 0.015 & 14.216 & 0.016 \\
\hline \hline
\end{tabular}


Table 16. $B V R I$ photometry of SN 2013dy, given in the Vega-system

\begin{tabular}{|c|c|c|c|c|c|c|c|c|}
\hline MJD & B & $\sigma_{B}$ & $\mathrm{~V}$ & $\sigma_{V}$ & $\mathrm{R}$ & $\sigma_{R}$ & I & $\sigma_{I}$ \\
\hline 56490.9 & 14.069 & 0.008 & 13.824 & 0.033 & 13.653 & 0.045 & 13.522 & 0.031 \\
\hline 56492.0 & 13.929 & 0.021 & 13.636 & 0.032 & 13.459 & 0.064 & 13.341 & 0.065 \\
\hline 56493.0 & 13.720 & 0.093 & 13.479 & 0.022 & 13.307 & 0.062 & 13.205 & 0.040 \\
\hline 56494.0 & 13.584 & 0.067 & 13.359 & 0.011 & 13.196 & 0.070 & 13.103 & 0.038 \\
\hline 56494.9 & 13.492 & 0.028 & 13.271 & 0.002 & 13.118 & 0.059 & 13.042 & 0.039 \\
\hline 56495.9 & 13.388 & 0.064 & 13.168 & 0.038 & 13.050 & 0.067 & 12.980 & 0.055 \\
\hline 56496.9 & 13.376 & 0.052 & 13.118 & 0.041 & 12.998 & 0.088 & 12.955 & 0.038 \\
\hline 56497.9 & 13.325 & 0.045 & 13.036 & 0.066 & 12.954 & 0.057 & 12.950 & 0.030 \\
\hline 56498.8 & 13.282 & 0.027 & 13.028 & 0.022 & 12.934 & 0.051 & 12.957 & 0.042 \\
\hline 56499.8 & 13.274 & 0.076 & 13.043 & 0.002 & 12.902 & 0.063 & 12.970 & 0.058 \\
\hline 56500.8 & 13.316 & 0.002 & 12.981 & 0.021 & 12.913 & 0.068 & 12.983 & 0.043 \\
\hline 56501.8 & 13.255 & 0.002 & 12.957 & 0.034 & 12.874 & 0.063 & 13.010 & 0.048 \\
\hline 56505.8 & 13.415 & 0.010 & 13.006 & 0.040 & 12.939 & 0.059 & 13.135 & 0.055 \\
\hline 56506.9 & 13.427 & 0.030 & 13.044 & 0.030 & 12.960 & 0.065 & 13.203 & 0.026 \\
\hline 56507.9 & 13.489 & 0.044 & 13.070 & 0.048 & 13.004 & 0.075 & 13.250 & 0.049 \\
\hline 56509.8 & 13.610 & 0.064 & 13.153 & 0.019 & 13.112 & 0.061 & 13.369 & 0.059 \\
\hline 56511.8 & 13.748 & 0.074 & 13.268 & 0.029 & 13.248 & 0.069 & 13.499 & 0.060 \\
\hline 56512.8 & 13.827 & 0.068 & 13.335 & 0.033 & 13.332 & 0.054 & 13.569 & 0.034 \\
\hline 56520.9 & 14.717 & 0.060 & 13.801 & 0.012 & 13.622 & 0.051 & 13.614 & 0.045 \\
\hline 56521.9 & 14.819 & 0.039 & 13.849 & 0.058 & 13.652 & 0.055 & 13.588 & 0.054 \\
\hline 56534.8 & 15.934 & 0.001 & 14.382 & 0.045 & 13.972 & 0.064 & 13.528 & 0.030 \\
\hline 56536.8 & 16.040 & 0.013 & 14.518 & 0.031 & 14.089 & 0.043 & 13.619 & 0.048 \\
\hline 56538.8 & 16.127 & 0.040 & 14.624 & 0.016 & 14.198 & 0.073 & 13.750 & 0.028 \\
\hline 56539.8 & 16.166 & 0.043 & 14.677 & 0.007 & 14.265 & 0.053 & 13.808 & 0.027 \\
\hline 56541.8 & 16.215 & 0.024 & 14.782 & 0.044 & 14.389 & 0.065 & 13.928 & 0.053 \\
\hline 56542.8 & 16.271 & 0.047 & 14.843 & 0.018 & 14.433 & 0.068 & 13.998 & 0.046 \\
\hline 56554.9 & 16.465 & 0.038 & 15.236 & 0.017 & 14.898 & 0.052 & 14.621 & 0.059 \\
\hline 56563.0 & 16.568 & 0.022 & 15.473 & 0.036 & 15.167 & 0.046 & 14.980 & 0.044 \\
\hline 56566.8 & 16.598 & 0.035 & 15.559 & 0.025 & 15.288 & 0.062 & 15.160 & 0.035 \\
\hline 56573.8 & 16.701 & 0.054 & 15.760 & 0.023 & 15.513 & 0.053 & 15.424 & 0.043 \\
\hline 56577.8 & 16.730 & 0.042 & 15.866 & 0.027 & 15.625 & 0.059 & 15.588 & 0.014 \\
\hline 56590.9 & 16.905 & 0.061 & 16.182 & 0.028 & 16.021 & 0.067 & 16.073 & 0.046 \\
\hline 56591.9 & 16.912 & 0.018 & 16.203 & 0.043 & 16.052 & 0.050 & 16.099 & 0.027 \\
\hline 56596.7 & 16.973 & 0.042 & 16.328 & 0.012 & 16.200 & 0.035 & 16.294 & 0.030 \\
\hline 56603.9 & 17.017 & 0.054 & 16.584 & 0.026 & 16.424 & 0.064 & 16.540 & 0.066 \\
\hline
\end{tabular}


VINKÓ ET AL.

Table 17. $g^{\prime} r^{\prime} i^{\prime} z^{\prime}$ photometry of SN 2013dy, given as AB-magnitudes

\begin{tabular}{|c|c|c|c|c|c|c|c|c|}
\hline MJD & $\mathrm{g}$ & $\sigma_{g}$ & $\mathrm{r}$ & $\sigma_{r}$ & $\mathrm{i}$ & $\sigma_{i}$ & $\mathrm{z}$ & $\sigma_{z}$ \\
\hline 56490.92 & 13.901 & 0.079 & 13.689 & 0.060 & 13.789 & 0.050 & 13.901 & 0.131 \\
\hline 56491.90 & 13.705 & 0.110 & 13.481 & 0.057 & 13.557 & 0.078 & 13.607 & 0.121 \\
\hline 56492.91 & 13.623 & 0.101 & 13.332 & 0.068 & 13.365 & 0.086 & 13.527 & 0.115 \\
\hline 56493.91 & 13.534 & 0.124 & 13.198 & 0.041 & 13.320 & 0.047 & 13.463 & 0.074 \\
\hline 56494.91 & 13.462 & 0.115 & 13.174 & 0.051 & 13.313 & 0.063 & 13.440 & 0.083 \\
\hline 56495.88 & 13.377 & 0.130 & 13.100 & 0.056 & 13.271 & 0.068 & 13.364 & 0.079 \\
\hline 56496.84 & 13.176 & 0.103 & 13.036 & 0.094 & 13.257 & 0.092 & 13.473 & 0.203 \\
\hline 56497.85 & 13.349 & 0.134 & 13.022 & 0.050 & 13.272 & 0.065 & 13.336 & 0.121 \\
\hline 56498.89 & 13.170 & 0.104 & 12.924 & 0.079 & 13.201 & 0.074 & 13.237 & 0.080 \\
\hline 56502.90 & 13.036 & 0.061 & 12.980 & 0.063 & 13.237 & 0.119 & 13.335 & 0.138 \\
\hline 56503.93 & 13.017 & 0.070 & 12.888 & 0.032 & 13.366 & 0.045 & 13.322 & 0.108 \\
\hline 56504.88 & 13.120 & 0.082 & 12.882 & 0.036 & 13.373 & 0.059 & 13.330 & 0.099 \\
\hline 56505.88 & 12.998 & 0.058 & 12.895 & 0.041 & 13.449 & 0.044 & 13.334 & 0.088 \\
\hline 56508.88 & 13.160 & 0.068 & 13.005 & 0.038 & 13.558 & 0.079 & 13.605 & 0.108 \\
\hline 56509.84 & 13.217 & 0.071 & 13.112 & 0.048 & 13.587 & 0.077 & 13.579 & 0.116 \\
\hline 56510.86 & 13.209 & 0.048 & 13.175 & 0.033 & 13.726 & 0.041 & 13.600 & 0.155 \\
\hline 56511.84 & 13.426 & 0.083 & 13.222 & 0.053 & 13.745 & 0.064 & 13.656 & 0.134 \\
\hline 56512.85 & 13.394 & 0.064 & 13.315 & 0.031 & 13.872 & 0.054 & 13.842 & 0.136 \\
\hline 56513.85 & 13.622 & 0.085 & 13.416 & 0.051 & 13.938 & 0.102 & 13.670 & 0.085 \\
\hline 56515.86 & 13.520 & 0.050 & 13.496 & 0.032 & 14.060 & 0.050 & 13.651 & 0.083 \\
\hline 56519.83 & 14.033 & 0.108 & 13.608 & 0.045 & 14.033 & 0.074 & 13.647 & 0.096 \\
\hline 56520.83 & 14.096 & 0.097 & 13.617 & 0.053 & 14.086 & 0.065 & 13.736 & 0.143 \\
\hline 56521.82 & 14.133 & 0.091 & 13.660 & 0.055 & 14.036 & 0.064 & 13.644 & 0.124 \\
\hline 56526.82 & 14.525 & 0.159 & 13.803 & 0.088 & 13.916 & 0.097 & 13.582 & 0.087 \\
\hline 56530.87 & 14.663 & 0.067 & 13.826 & 0.054 & 13.913 & 0.053 & 13.580 & 0.125 \\
\hline 56534.87 & 14.812 & 0.061 & 13.959 & 0.029 & 13.977 & 0.035 & 13.640 & 0.100 \\
\hline 56535.79 & 14.929 & 0.077 & 14.060 & 0.046 & 14.121 & 0.096 & 13.792 & 0.215 \\
\hline 56538.82 & 15.068 & 0.053 & 14.214 & 0.044 & 14.200 & 0.061 & 13.836 & 0.112 \\
\hline 56539.81 & 15.108 & 0.067 & 14.252 & 0.033 & 14.261 & 0.043 & 13.921 & 0.126 \\
\hline 56541.79 & 15.152 & 0.051 & 14.348 & 0.035 & 14.371 & 0.059 & 13.991 & 0.126 \\
\hline 56542.83 & 15.225 & 0.057 & 14.380 & 0.031 & 14.412 & 0.064 & 14.071 & 0.126 \\
\hline 56543.83 & 15.377 & 0.118 & 14.487 & 0.051 & 14.463 & 0.067 & 14.187 & 0.098 \\
\hline 56551.76 & 15.989 & 0.190 & 14.911 & 0.082 & 15.037 & 0.113 & 14.768 & 0.153 \\
\hline 56552.80 & 15.496 & 0.103 & 14.731 & 0.054 & 14.866 & 0.077 & 14.616 & 0.123 \\
\hline 56554.83 & 15.782 & 0.162 & 14.813 & 0.043 & 14.900 & 0.052 & 14.880 & 0.101 \\
\hline 56557.79 & 15.536 & 0.100 & 14.899 & 0.062 & 14.908 & 0.075 & 14.920 & 0.150 \\
\hline 56558.90 & 15.572 & 0.085 & 14.879 & 0.036 & 15.002 & 0.053 & 14.842 & 0.099 \\
\hline 56559.77 & 15.496 & 0.066 & 14.939 & 0.033 & 15.049 & 0.046 & 15.022 & 0.142 \\
\hline 56560.76 & 15.517 & 0.078 & 14.978 & 0.052 & 15.071 & 0.068 & 15.149 & 0.207 \\
\hline 56568.79 & 15.570 & 0.068 & 15.117 & 0.042 & 15.269 & 0.063 & 15.257 & 0.082 \\
\hline 56569.77 & 15.636 & 0.066 & 15.188 & 0.058 & 15.240 & 0.064 & 15.241 & 0.135 \\
\hline 56575.91 & 15.752 & 0.074 & 15.262 & 0.049 & 15.444 & 0.067 & 15.529 & 0.129 \\
\hline 56578.73 & 15.815 & 0.095 & 15.325 & 0.046 & 15.479 & 0.055 & 15.537 & 0.116 \\
\hline 56582.90 & 16.002 & 0.110 & 15.504 & 0.062 & 15.520 & 0.068 & 15.640 & 0.119 \\
\hline 56584.74 & 15.941 & 0.132 & 15.432 & 0.055 & 15.952 & 0.131 & 15.511 & 0.131 \\
\hline 56586.78 & 15.976 & 0.102 & 15.548 & 0.058 & 15.726 & 0.074 & 15.618 & 0.108 \\
\hline 56588.81 & 15.836 & 0.091 & 15.538 & 0.058 & 15.659 & 0.060 & 15.863 & 0.136 \\
\hline 56590.80 & 15.880 & 0.081 & 15.650 & 0.069 & 15.705 & 0.061 & 15.781 & 0.139 \\
\hline 56591.77 & 15.842 & 0.061 & 15.533 & 0.038 & 15.645 & 0.059 & 15.759 & 0.109 \\
\hline 56594.73 & 15.907 & 0.048 & 15.652 & 0.050 & 15.743 & 0.063 & 16.255 & 0.201 \\
\hline 56595.98 & 16.008 & 0.092 & 15.909 & 0.129 & 15.789 & 0.102 & 16.586 & 0.356 \\
\hline 56597.97 & 15.893 & 0.134 & 15.911 & 0.126 & 15.958 & 0.182 & 16.909 & 0.590 \\
\hline 56603.75 & 15.991 & 0.052 & 15.709 & 0.037 & 15.882 & 0.052 & 15.871 & 0.103 \\
\hline 56627.82 & 16.224 & 0.072 & 16.012 & 0.058 & 16.025 & 0.076 & 16.152 & 0.180 \\
\hline 56628.82 & 16.319 & 0.085 & 16.127 & 0.089 & 16.120 & 0.084 & 15.890 & 0.149 \\
\hline
\end{tabular}

Table 18. Local $B V R I$ standard stars in the vicinity of SN 2014J

\begin{tabular}{cccccccccc}
\hline \hline R.A. & Dec. & $\mathrm{B}$ & $\sigma_{B}$ & $\mathrm{~V}$ & $\sigma_{V}$ & $\mathrm{R}$ & $\sigma_{R}$ & $\mathrm{I}$ & $\sigma_{I}$ \\
\hline 09:56:37.96 & $+69: 41: 18.9$ & 15.024 & 0.012 & 14.277 & 0.010 & 13.855 & 0.007 & 13.396 & 0.010 \\
09:56:32.99 & $+69: 39: 17.9$ & 13.983 & 0.008 & 13.504 & 0.007 & 13.209 & 0.005 & 12.816 & 0.008 \\
\hline \hline
\end{tabular}


Table 19. Photometry of SN 2014J. The $B V R I$ data are given in Vega-magnitudes, while the $g^{\prime} r^{\prime} i^{\prime} z^{\prime}$ data are in AB-magnitudes.

\begin{tabular}{|c|c|c|c|c|c|c|c|c|}
\hline MJD & B & $\sigma_{B}$ & $\mathrm{v}$ & $\sigma_{V}$ & $\mathrm{R}$ & $\sigma_{R}$ & I & $\sigma_{I}$ \\
\hline 56684.0 & 12.142 & 0.040 & 10.921 & 0.018 & 10.282 & 0.003 & 9.802 & 0.031 \\
\hline 56688.8 & 11.846 & 0.068 & 10.669 & 0.015 & 10.093 & 0.028 & 9.697 & 0.002 \\
\hline 56691.0 & 11.971 & 0.117 & 10.622 & 0.025 & 10.040 & 0.008 & 9.827 & 0.004 \\
\hline 56692.0 & 11.931 & 0.081 & 10.588 & 0.037 & 10.071 & 0.015 & 9.858 & 0.020 \\
\hline 56693.0 & 11.965 & 0.032 & 10.584 & 0.031 & 10.078 & 0.027 & 9.819 & 0.011 \\
\hline 56703.0 & 12.818 & 0.020 & 11.136 & 0.022 & 10.655 & 0.013 & 10.367 & 0.005 \\
\hline 56706.2 & 13.211 & 0.071 & 11.345 & 0.041 & 10.835 & 0.018 & 10.348 & 0.027 \\
\hline 56709,0 & 13.538 & 0.085 & 11.362 & 0.071 & 10.770 & 0.037 & 10.280 & 0.078 \\
\hline 56712.0 & 13.745 & 0.020 & 11.458 & 0.027 & 10.789 & 0.007 & 10.122 & 0.004 \\
\hline 56717.8 & 14.521 & 0.001 & 11.726 & 0.002 & 10.995 & 0.004 & 10.184 & 0.012 \\
\hline 56724.9 & 14.822 & 0.024 & 12.179 & 0.021 & 11.326 & 0.009 & 10.425 & 0.010 \\
\hline 56726.0 & 14.944 & 0.035 & 12.152 & 0.026 & 11.371 & 0.014 & 10.480 & 0.015 \\
\hline 56727.0 & 15.009 & 0.040 & 12.250 & 0.008 & 11.457 & 0.003 & 10.545 & 0.019 \\
\hline 56728.0 & 14.969 & 0.006 & 12.343 & 0.040 & 11.537 & 0.018 & 10.632 & 0.020 \\
\hline 56729.0 & 15.060 & 0.006 & 12.367 & 0.041 & 11.550 & 0.001 & 10.687 & 0.030 \\
\hline 56730.9 & 15.003 & 0.002 & 12.438 & 0.019 & 11.650 & 0.023 & 10.643 & 0.008 \\
\hline 56736.9 & 15.042 & 0.004 & 12.659 & 0.006 & 11.904 & 0.005 & 11.148 & 0.015 \\
\hline 56739.0 & 15.124 & 0.017 & 12.714 & 0.016 & 11.958 & 0.043 & 11.034 & 0.024 \\
\hline 56741.9 & 15.169 & 0.018 & 12.792 & 0.019 & 12.035 & 0.002 & 11.238 & 0.005 \\
\hline 56742.8 & 15.156 & 0.001 & 12.809 & 0.001 & 12.065 & 0.001 & 11.371 & 0.001 \\
\hline 56744.8 & 15.142 & 0.026 & 12.855 & 0.002 & 11.920 & 0.014 & 11.370 & 0.012 \\
\hline 56746.0 & 15.198 & 0.017 & 12.916 & 0.022 & 12.092 & 0.005 & 11.411 & 0.011 \\
\hline 56746.9 & 15.200 & 0.001 & 12.938 & 0.007 & 12.060 & 0.007 & 11.480 & 0.009 \\
\hline 56751.8 & 15.223 & 0.019 & 13.045 & 0.018 & 12.318 & 0.008 & 11.711 & 0.025 \\
\hline 56754.8 & 15.248 & 0.030 & 13.124 & 0.008 & 12.420 & 0.007 & 11.790 & 0.006 \\
\hline 56774.8 & 15.215 & 0.029 & 13.602 & 0.004 & 12.945 & 0.007 & 12.480 & 0.013 \\
\hline 56777.9 & 15.407 & 0.008 & 13.682 & 0.021 & 13.062 & 0.004 & 12.608 & 0.035 \\
\hline 56785.8 & 15.405 & 0.043 & 13.829 & 0.002 & 13.199 & 0.010 & 12.731 & 0.008 \\
\hline 56798.9 & 15.571 & 0.014 & 14.108 & 0.020 & 13.545 & 0.007 & 13.078 & 0.02 .5 \\
\hline MJD & $\mathrm{g}$ & $\sigma_{g}$ & $\mathrm{r}$ & $\sigma_{r}$ & $\mathrm{i}$ & $\sigma_{i}$ & $\mathrm{z}$ & $\sigma_{z}$ \\
\hline 56680.05 & 12.295 & 0.030 & 10.941 & 0.056 & 10.836 & 0.023 & 10.415 & 0.034 \\
\hline 56682.97 & 11.733 & 0.033 & 10.554 & 0.063 & 10.397 & 0.013 & 10.072 & 0.037 \\
\hline 56683.97 & 11.582 & 0.018 & 10.504 & 0.081 & 10.281 & 0.033 & 9.872 & 0.058 \\
\hline 56691.07 & 11.267 & 0.014 & 10.213 & 0.012 & 10.311 & 0.019 & 9.977 & 0.029 \\
\hline 56692.10 & 11.278 & 0.016 & 10.199 & 0.007 & 10.340 & 0.016 & 10.018 & 0.032 \\
\hline 56692.97 & 11.284 & 0.011 & 10.218 & 0.012 & 10.354 & 0.016 & 10.044 & 0.038 \\
\hline 56698.12 & 11.414 & 0.016 & 10.435 & 0.004 & 10.610 & 0.026 & 10.172 & 0.024 \\
\hline 56700.98 & 11.540 & 0.017 & 10.694 & 0.020 & 10.967 & 0.046 & 10.269 & 0.033 \\
\hline 56701.79 & 11.627 & 0.010 & 10.719 & 0.010 & 10.908 & 0.031 & 10.254 & 0.033 \\
\hline 56702.97 & 11.687 & 0.012 & 10.787 & 0.011 & 10.970 & 0.028 & 10.267 & 0.039 \\
\hline 56703.76 & 11.718 & 0.005 & 10.851 & 0.006 & 10.970 & 0.023 & 10.259 & 0.052 \\
\hline 56713.90 & 12.509 & 0.017 & 10.958 & 0.010 & 10.821 & 0.032 & 10.075 & 0.037 \\
\hline 56714.84 & 12.518 & 0.015 & 10.990 & 0.014 & 10.749 & 0.021 & 10.104 & 0.034 \\
\hline 56715.77 & 12.572 & 0.029 & 10.987 & 0.017 & 10.734 & 0.019 & 10.091 & 0.062 \\
\hline 56717.85 & 12.697 & 0.012 & 11.069 & 0.011 & 10.815 & 0.030 & 10.124 & 0.038 \\
\hline 56719.91 & 12.879 & 0.011 & 11.134 & 0.010 & 10.878 & 0.023 & 10.116 & 0.030 \\
\hline 56724.97 & 13.230 & 0.009 & 11.489 & 0.012 & 11.133 & 0.026 & 10.336 & 0.035 \\
\hline 56725.86 & 13.225 & 0.021 & 11.581 & 0.010 & 11.182 & 0.026 & 10.443 & 0.035 \\
\hline 56727.93 & 13.375 & 0.014 & 11.650 & 0.016 & 11.336 & 0.030 & 10.509 & 0.036 \\
\hline 56728.93 & 13.467 & 0.020 & 11.747 & 0.012 & 11.424 & 0.029 & 10.584 & 0.034 \\
\hline 56729.95 & 13.473 & 0.027 & 11.765 & 0.016 & 11.489 & 0.035 & 10.620 & 0.030 \\
\hline 56730.80 & 13.400 & 0.022 & 11.807 & 0.012 & 11.471 & 0.030 & 10.752 & 0.034 \\
\hline 56733.93 & 13.552 & 0.016 & 11.961 & 0.017 & 11.614 & 0.032 & 10.938 & 0.029 \\
\hline 56734.75 & 13.457 & 0.047 & 11.899 & 0.057 & 11.565 & 0.035 & 11.144 & 0.053 \\
\hline 56737.85 & 13.627 & 0.013 & 12.047 & 0.018 & 11.760 & 0.033 & 11.074 & 0.040 \\
\hline 56745.79 & 13.815 & 0.011 & 12.297 & 0.019 & 12.063 & 0.039 & 11.482 & 0.040 \\
\hline 56746.92 & 13.748 & 0.104 & 12.300 & 0.035 & 12.112 & 0.051 & 11.655 & 0.119 \\
\hline 56754.82 & 13.980 & 0.016 & 12.558 & 0.022 & 12.293 & 0.040 & 11.866 & 0.049 \\
\hline 56768.80 & 14.227 & 0.020 & 12.910 & 0.030 & 12.682 & 0.047 & 12.493 & 0.052 \\
\hline 56769.88 & 14.236 & 0.018 & 12.965 & 0.029 & 12.715 & 0.044 & 12.390 & 0.058 \\
\hline 56773.86 & 14.314 & 0.018 & 13.079 & 0.028 & 12.852 & 0.046 & 12.551 & 0.058 \\
\hline 56775.88 & 14.255 & 0.028 & 13.095 & 0.034 & 12.896 & 0.054 & 12.976 & 0.114 \\
\hline 56782.84 & 14.382 & 0.024 & 13.294 & 0.029 & 13.037 & 0.052 & 12.750 & 0.058 \\
\hline 56783.84 & 14.464 & 0.018 & 13.346 & 0.028 & 13.060 & 0.056 & 12.849 & 0.069 \\
\hline 56785.81 & 14.512 & 0.019 & 13.361 & 0.032 & 13.098 & 0.052 & 12.828 & 0.062 \\
\hline 56787.86 & 14.547 & 0.022 & 13.384 & 0.032 & 13.114 & 0.055 & 12.887 & 0.069 \\
\hline 56796.91 & 14.654 & 0.029 & 13.628 & 0.049 & 13.388 & 0.068 & 13.056 & 0.081 \\
\hline 56797.88 & 14.672 & 0.025 & 13.604 & 0.049 & 13.312 & 0.068 & 13.033 & 0.08. \\
\hline 56798.84 & 14.670 & 0.029 & 13.603 & 0.043 & 13.245 & 0.057 & 12.918 & 0.068 \\
\hline 56799.89 & 14.686 & 0.022 & 13.633 & 0.037 & 13.305 & 0.060 & 13.097 & 0.076 \\
\hline 56805.87 & 14.834 & 0.028 & 13.747 & 0.054 & 13.362 & 0.077 & 13.156 & 0.084 \\
\hline 56811.86 & 14.898 & 0.035 & 13.893 & 0.052 & 13.530 & 0.072 & 13.187 & 0.088 \\
\hline
\end{tabular}

